\title{
Distributed Optimal State Consensus for Multiple Circuit Systems with Disturbance Rejection
}

\author{
Zhu Wang, Dong Wang, and Dongbing Gu
}

\begin{abstract}
This paper investigates the distributed optimal state consensus problem for an electronic system with a group of circuit units. The dynamics of each unit is modeled by a Chua's circuit in the presence of disturbance generated by an external system. By means of the internal model approach and feedback control, a compensator-based continuous-time algorithm is proposed to minimize the sum of all cost functions associated with each individual unit in a cooperative manner. Supported by convex analysis, graph theory and Lyapunov theory, it is proved that the proposed algorithm is exponentially convergent. Compared with the centralized algorithms, the proposed protocol possesses remarkable superiority in improving scalability and reliability of multiple circuit systems. Moreover, we also study the distributed uncertain optimal state consensus problem and a linear regret bound is obtained in this case. Finally, a state synchronization example is provided to validate the effectiveness of the proposed algorithms.
\end{abstract}

Index Terms-Distributed optimization, consensus control, disturbance rejection, internal model (IM), regret bound.

\section{INTRODUCTION}

$\mathrm{D}$ ISTRIBUTED coordination for large-scale networked systems with many units has attracted increasing attentions owing to its extensive applications. In these systems, units can interact with each other via a communication network to perform complex tasks, e.g., consensus, formation control, resource allocation and optimization [1]-[4].

Consensus is an important issue for dynamical networks. Its objective is to develop an appropriate control law making use of local information for each unit such that states of all units converge to a common value [5]. For example, [6]-[8] studied the state consensus problem of a nonlinear circuit network by using feedback control method. In [9], [10], the authors proposed novel driving approaches to compelling that the states of Chua's circuit system achieve consensus. With the help of matrix theory and Lyapunov methods, some consensus criteria were obtained to guarantee that the states of a coupled nonlinear circuit system achieve consensus in [11]. Zhang and Feng [12] studied the distributed output regulation problem of piecewise discrete-time systems via an error feedback method, and the obtained results were further applied to the consensus problem of multiple circuit networks. Angelo et al. [13] studied the consensus problem of nonlinear periodic circuits via a small-signal analysis method. In addition, distributed consensus for multi-agent systems with uncertainties has been studied in the past several years. For example, Wen et al. [14] proposed an adaptive consensus-based algorithm for solving the economic dispatch problem of smart grids subject to communication uncertainties. Diwadkar and Vaidya

- Z. Wang and D. Wang are with the Key Laboratory of Intelligent Control and Optimization for Industrial Equipment of Ministry of Education, and with the School of Control Science and Engineering, Dalian University of Technology, Dalian, 116024 China.

E-mail: xinyu306@mail.dlut.edu.cn, dwang@dlut.edu.cn.

- D. Gu is with the School of Computer Science and Electronic Engineering, University of Essex, Colchester, C04 3SQ, U.K.

E-mail:dgu@essex.ac.uk.
[15] considered the consensus problem for a large-scale nonlinear system in the presence of uncertain communication links, and some consensus conditions were also derived with the aid of a random version of Positive Real Lemma.

When the consistent state is required to minimize the sum of a group of convex cost functions known by each individual unit, then the consensus problem evolves into a distributed optimization problem in [16]. For such a collaborative problem, we here call it the distributed optimal state consensus problem (DOSCP). To solve such a problem, many results have been reported, see [17]-[24]. Some results about discrete-time algorithms have been developed to solve the DOSCP. For example, the DOSCP was first studied in [17]. A primal-dual subgradient method was developed to solve the online optimization problem in [24]. There is also growing interest to explore more suitable continuoustime algorithms to solve the DOSCP. For instance, the seminal work [19] provided a class of novel algorithms to deal with the DOSCP from a control viewpoint, and the class of continuous-time algorithms was further improved to solve the DOSCP in [20]. The DOSCP for nonlinear systems was considered in [21]. In [22], the DOSCP for nonlinear systems was solved by gradient-based algorithms. A time-varying distributed optimization problem for a single/double integrator system was illustrated in [23].

The dynamics of some networked systems are described as integral-type systems, such as [17]-[20], [23]. However, in many applications, the dynamics of physical systems are more complex such as mechanical systems, power systems, circuit systems, etc. Solving the DOSCP for more complex systems is more challenging and demanding. The cost function is time-invariant in the aforementioned distributed optimization works [17]-[22]. The time-varying cost function is considered in [23], [24]. However, although they have demonstrated some positive results, there are still many topics to be solved such as online optimization and trajectory optimization. In addition, when the circuit units operate, they are often affected by various distur- 
bances resulting from communication or environment [21]. Disturbance rejection becomes an urgent problem to be solved. Many published results can only guarantee that the system's final state converges to a small neighborhood of the consensus point under the existence of disturbances [25], [26], but can not achieve the consensus with more accuracy. An effective approach to disturbance rejection has been developed based on the internal model principle from the viewpoint of output regulation in [27]. With regard to distributed optimization, an internal model-based control law was designed to solve the DOSCP with disturbance rejection in [21]. However, to the best of our knowledge, there are very few results related to the DOSCP for nonlinear circuit systems with higher relative degree and disturbance rejection, which motivates the present exploration.

Our goal in this paper is to further study the DOCSP for nonlinear circuit systems with disturbance rejection. It is worth noting that, compared with the existing results, the distributed optimal state consensus problem under consideration is quite comprehensive. Specifically, we consider the DOSCP with not only the deterministic cost function but also the uncertain cost function. The main contributions of this paper are emphasized as follows: (i) We formulate a DOSCP for a nonlinear electronic system to illustrate how a group of circuit units cooperatively demonstrate their performance on state consensus. The consistent state is subject to the minimum value of the cost function of entire system. Furthermore, this formulation can be regarded as an extension of some well-known problems such as the DOP in [19], [20]. We focus on the nonlinear dynamics with high relative degree under the consideration of external disturbance, which is different from the existing integral-type and linear systems in the design of continuous-time controllers [17]-[20]. (ii) A novel algorithm based on feedback control and internal model techniques is developed to tackle the technical challenges brought by external disturbances and nonlinear dynamics. With the help of graph theory, convex analysis and Lyapunov theory, it is shown that the proposed algorithm converges exponentially to the optimal solution of the considered problem. (iii) The distributed uncertain optimal state consensus problem with inaccurate gradients is illustrated and a linear regret bound is obtained in this case as well.

Organization. In Section 2, notations and some preliminaries are given, followed by problem formulation. In Section 3, the DOSCP with disturbance rejection is studied. Then the uncertain DOSCP with disturbance rejection is illustrated in Section 4. An example is provided to demonstrate the effectiveness of the proposed algorithms in Section 5. Section 6 summarises the investigation.

Notations. Throughout this paper, $Q$ and $Q^{T}$ denote a real matrix and its transpose, respectively. $\mathbb{R}$ and $\mathbb{R}^{n}$ denote the set of the real numbers and the space of the real vectors with $n$ dimension, respectively. $\mathbb{R}^{+}$denotes the set of the positive integers. $1_{n}$ is a $n$-dimensional vector with all elements $1 . I_{n}$ is the identity matrix of $n$ dimension. $\operatorname{diag}\left\{x_{1}, x_{2}, \ldots, x_{n}\right\}$ denotes a diagonal matrix with entries $x_{i}, i=1,2, \ldots, n$. Denote by $\operatorname{col}\left(x_{1}, x_{2}, \ldots, x_{n}\right)=$ $\left[x_{1}^{T}, x_{2}^{T}, \ldots, x_{n}^{T}\right]^{T}$ the column vector stacked with vectors $x_{1}, x_{2}, \ldots, x_{n} \cdot\|\cdot\|$ denotes the standard Euclidean norm.

\section{PRELIMINARIES AND PROBLEM FORMULATION}

\subsection{Convex Analysis}

A nonempty set $S \in \mathbb{R}^{n}$ is convex if $b z+(1-b) z_{1} \in S$, $\forall z, z_{1} \in S$ and $b \in[0,1]$. A function $f: S \rightarrow \mathbb{R}$ is convex over the convex set $S$ if $f\left(b z+(1-b) z_{1}\right) \leq$ $b f(z)+(1-b) f\left(z_{1}\right), \forall b \in[0,1]$ and $z, z_{1} \in S$. A differentiable function $f: S \rightarrow \mathbb{R}$ is strongly convex over $S$ if $\left(z-z_{1}\right)^{T}\left(\nabla f(z)-\nabla f\left(z_{1}\right)\right) \geq \hat{w}\left\|z-z_{1}\right\|^{2}$ with a positive constant $\hat{w}, \forall z, z_{1} \in S$. Moreover, its gradient $\nabla f$ is Lipschitz over $S$ if $\left\|\nabla f(z)-\nabla f\left(z_{1}\right)\right\| \leq r\left\|z-z_{1}\right\|, \forall z, z_{1} \in$ $S$, where $r$ is the Lipschitz constant, see [28].

\subsection{Problem Formulation}

We consider a state consensus problem for an electronic system consisting of $n$ circuit units. Suppose that each unit is equipped with a sensor for exchanging information with its neighbor units to complete a complex task, and it has the ability to perform independent computation. All units collaboratively find an unknown agreement value that enables the state of the entire system to be consensus. The circuit unit to be considered is shown in Fig. 1. It is called a Chua's circuit in [29], [30] disturbed by external disturbances that can be regarded as the effect of the working environment on the system's performance. Furthermore, the new circuit model can be described by the following differentiable equations:

$$
\begin{aligned}
C_{1} \dot{v}_{1 i}(t)= & \frac{v_{2 i}(t)-v_{1 i}(t)}{R}-f\left(v_{1 i}(t)\right)+\frac{C_{1} B_{0} G}{C_{2}} u_{i}(t) \\
& +\frac{C_{1} B_{0} G}{C_{2}} \theta_{i}(t), \\
C_{2} \dot{v}_{2 i}(t)= & \frac{v_{1 i}(t)-v_{2 i}(t)}{R}-w_{i}(t), \\
L \dot{w}_{i}(t)= & v_{2 i}(t)-R_{0} w_{i}(t),
\end{aligned}
$$

where $w_{i}(t)$ denotes the current across the inductor $L . v_{1 i}(t)$ and $v_{2 i}(t)$ are the voltages through the capacitors $C_{1}$ and $C_{2}$, respectively. $u_{i}(t)$ is the current from the external power source as active control action of unit $i$ to compel $w_{i}(\mathrm{t})$, $v_{1 i}(t)$ and $v_{2 i}(t)$ for achieving consensus. $R$ and $R_{0}$ are linear resistors, $G=1 / R, f\left(v_{1 i}(t)\right)$ is the $v-i$ property of the nonlinear conductor $N_{r}$ as shown in Fig. 2 with a slope $G_{a}$ in the inner region and $G_{b}$ in the outer region. The breakdown voltage $B_{0}$ of Chua's diode, i.e., $f\left(v_{1 i}(t)\right)=$ $G_{b} v_{1 i}(t)-0.5\left(G_{a}-G_{b}\right)\left(\left|v_{1 i}(t)+B_{0}\right|-\left|v_{1 i}(t)-B_{0}\right|\right)$ and

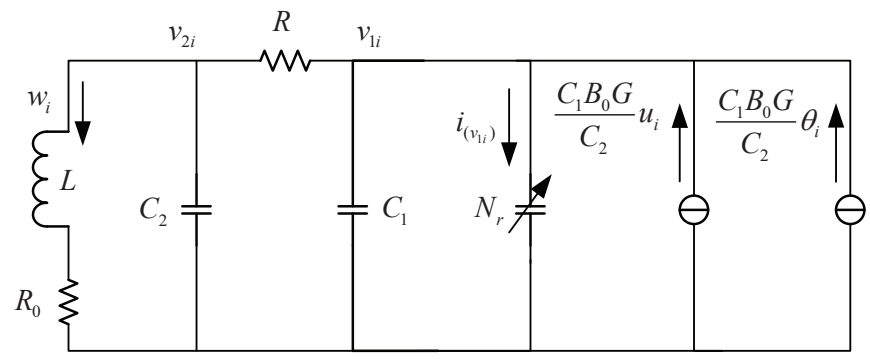

Fig. 1. The circuit structure of each unit. 


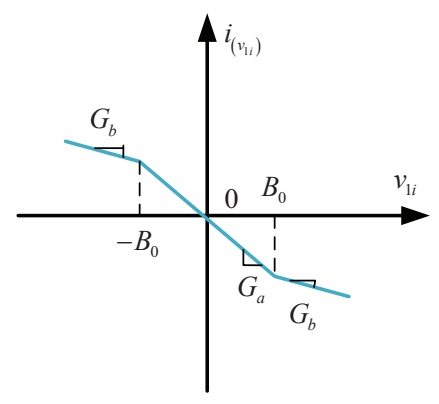

Fig. 2. The $v-i$ property of the nonlinear conductor $N_{r}$.

$\theta_{i}$ is a local disturbance generated by the following autonomous system

$$
\dot{\nu}_{i}=F \nu_{i}, \nu_{i}(0) \in \mathbb{V}_{i},
$$

where $\nu_{i}$ is the state, $\mathbb{V}_{i}$ is a fixed compact set, $F$ is a known matrix and its all eigenvalues are located on the imaginary axis of the complex plane [27], $i=1,2, \ldots, n$.

For the convenience of analysis, let $x_{i 1}=\frac{w_{i}}{B_{0} G}, x_{i 2}=$ $\frac{v_{i 2}}{B_{0}}, q_{i}=\frac{v_{i 1}}{B_{0}}, d_{i 1}=\frac{C_{2}}{C_{1}}, d_{i 2}=\frac{G_{a}}{G}, d_{i 3}=\frac{G_{b}}{G}, d_{i 4}=$ $\frac{C_{2}}{L G^{2}}, d_{i 5}=\frac{C_{2} R_{0}}{L G}, t^{\prime}=\frac{G t}{C_{2}}$, thus the dynamical behavior (1) of unit $i$ can be simplified to the following form

$$
\begin{aligned}
\dot{q}_{i}= & -d_{i 5} q_{i}+d_{i 4} x_{i 1}, \\
\dot{x}_{i 1}= & x_{i 2}-x_{i 1}-q_{i}, \\
\dot{x}_{i 2}=d_{i 1}\left[-0.5\left(d_{i 2}-d_{i 3}\right)\left(\left|x_{i 2}+1\right|-\left|x_{i 2}-1\right|\right)\right. & \left.\quad+x_{i 1}-\left(1+d_{i 3}\right) x_{i 2}\right]+u_{i}+\theta_{i} .
\end{aligned}
$$

Furthermore, let $y_{i}=x_{i 1}, x_{i}=x_{i 2}-x_{i 1}-q_{i}, h_{i}^{\prime}=$ $\operatorname{col}\left(d_{i 1}, d_{i 2}, d_{i 3}, d_{i 4}, d_{i 5}\right)$, then system (3) can be equivalently described by the following uncertain nonlinear state space equation with local disturbances

$$
\begin{aligned}
& \dot{q}_{i}=v_{i 1}\left(q_{i}, y_{i}, h_{i}^{\prime}\right), \\
& \dot{y}_{i}=x_{i}, \\
& \dot{x}_{i}=v_{i 2}\left(q_{i}, y_{i}, x_{i}, h_{i}^{\prime}\right)+u_{i}+\theta_{i},
\end{aligned}
$$

where $v_{i 1}\left(q_{i}, y_{i}, h_{i}^{\prime}\right)=-d_{i 5} q_{i}+d_{i 4} y_{i}, v_{i 2}\left(q_{i}, y_{i}, x_{i}, h_{i}^{\prime}\right)=$ $-0.5 d_{i 1}\left(d_{i 2}-d_{i 3}\right)\left(\left|x_{i}+y_{i}+q_{i}+1\right|-\left|x_{i}+y_{i}+q_{i}-1\right|\right)+$ $d_{i 1}\left(1+d_{i 3}\right)\left(x_{i}+y_{i}+q_{i}\right)+d_{i 1} y_{i}-x_{i}-d_{i 5} q_{i}+d_{i 4} y_{i}$. $\left(q_{i}, x_{i}, y_{i}\right) \in \mathbb{R} \times \mathbb{R} \times \mathbb{R}$ are also viewed as states of unit $i$ under new coordinates, $i=1,2, . ., n . h_{i}^{\prime} \in \mathbb{R}^{5}$ is an uncertain constant parameter in a fixed compact set $W \in \mathbb{R}^{5} . v_{i 1}\left(q_{i}, y_{i}, h_{i}^{\prime}\right)$ and $v_{i 2}\left(q_{i}, y_{i}, x_{i}, h_{i}^{\prime}\right)$ are Lipschitz and sufficiently smooth functions. It is worth noting that, in real applications, the disturbance is inevitable due to the uncertainty of the working environment. Hence, the state $\nu_{i}$ of (2) is not directly obtained. Although $\theta_{i}$ in (4) denotes a local disturbance, it is uncertain. In this paper we see that $\theta_{i}$ can be defined as the output of (2), i.e., $\theta_{i}:=o\left(\nu_{i}, h_{i}^{\prime}\right)$, which is measurable by some specific equipments or instrument. Moreover, $\theta_{i}:=o\left(\nu_{i}, h_{i}^{\prime}\right)$ is a polynomial in $\nu_{i}$ with coefficients depending on the uncertain parameter $h_{i}^{\prime}$. A system with the dynamics of (4) represents a class of minimum-phase uncertain nonlinear systems with second relative degree that can model many physical systems such as Chua's circuit [22] and synchronous generators with an infinite bus [31] in the presence of external disturbance.
Since each unit has different states, it requires to move to a common point under the drive of the developed controller. In this process, each circuit unit needs to consume energy. The energy expenditure is modeled by a convex function related to its own partial state, i.e., $f_{i}\left(y_{i}\right): y_{i} \rightarrow \mathbb{R}$, which can represent the cost resulting from the energy consumed by each unit, and it is only known by this unit. Thus, the state consensus problem can be formulated as searching for a rendezvous point as estimate of the unknown consistent state by minimizing the cost function with an equality constraint. The cost function is the sum of all the local cost functions associated with each unit, i.e.,

$$
\begin{aligned}
& \min f(y)=\sum_{i=1}^{n} f_{i}\left(y_{i}\right), \\
& \text { subject to }\left\|y_{i}-y_{j}\right\|=0,
\end{aligned}
$$

where $y=\left[y_{1}, y_{2}, \ldots, y_{n}\right]^{T}$. Hence, the state consensus problem for the electronic system with $n$ circuit units can be treated as finding the minimizer of the cost function subject to an equality constraint. The optimal solution is viewed as estimate of the consistent state for the entire system. Here suppose that the optimal solution set $Y^{*}$ of (5) is nonempty.

\subsection{Network Model}

The communication between units is described by a graph $\mathcal{G}(\mathcal{V}, \mathcal{E})$, named communication graph in [32], where $\mathcal{V}$ is the set of circuit units and $\mathcal{E}$ is the set of edges. In this paper, only simple graphs are considered, namely, the communication topology contains neither self-loops nor multiple edges between any two distinct circuit units. A graph is connected if there exists a path consisting of a sequence of distinct edges from a circuit unit to any other circuit unit, otherwise disconnected. In order to describe the relationship among circuit units, the adjacency matrix $\mathcal{A}=\left[a_{i j}\right] \in \mathbb{R}^{n \times n}$ is used, where $a_{i j}=1$ if $(i, j) \in \mathcal{E}$ and $a_{i j}=0$, otherwise. Unit $j$ is called a neighbor of unit $i$ if units $i$ and $j$ are directly connected. The notation $N_{i}=\{j \mid(i, j) \in \mathcal{E}\}$ denotes the set of neighbors of unit $i$. The degree of unit $i$ is $D_{i}=\sum_{j=1}^{n} a_{i j}$. Thus, the Laplacian matrix of $\mathcal{G}$ is defined as $\mathcal{L}=\mathcal{D}-\mathcal{A}$ with $\mathcal{D}=\operatorname{diag}\left\{D_{1}, D_{2}, \ldots, D_{n}\right\}$.

The assumptions about the communication topology and the local cost function are provided below.

Assumption 1. The communication topology between circuit units is an undirected and connected graph.

Lemma 1 ( [33]). 1) Under Assumption 1, the Laplacian matrix $\mathcal{L}$ of the graph $\mathcal{G}$ is symmetric and positive semidefinite, which satisfies $1_{n}^{T} \mathcal{L}=0$ and $\mathcal{L} 1_{n}=0$.

2) 0 is a simple eigenvalue of $\mathcal{L}$ and other eigenvalues are positive.

3) There is a non-zero vector $\hat{x} \in \mathbb{R}^{n}$ satisfying

$$
\hat{\lambda}_{2}=\min _{1_{n}^{T} \hat{x}=0} \frac{\hat{x}^{T} \mathcal{L} \hat{x}}{\hat{x}^{T} \hat{x}}, \hat{\lambda}_{n}=\max _{\hat{x} \neq 0} \frac{\hat{x}^{T} \mathcal{L} \hat{x}}{\hat{x}^{T} \hat{x}},
$$

where $\hat{\lambda}_{2}$ and $\hat{\lambda}_{n}$ is the minimal non-zero and maximal eigenvalues of $\mathcal{L}$, respectively.

Assumption 2. The local cost function $f_{i}\left(y_{i}\right)$ is $m_{i}$-strongly convex and its gradient is $p_{i}$-Lipschitz, respectively. 
Remark 1. Assumption 2 is a basic condition in existing results [19], [20]. $f_{i}\left(y_{i}\right)$ is strongly convex, so is the global cost function $f(y)$, which can guarantee that the optimal solution of problem (5) is unique. It is further derived that $Y^{*}$ is a single set.

To proceed further, we introduce the following assumptions for nonlinear system (4).

Assumption 3. Given the point $y^{*} \in Y^{*}$, there exists a unique $q_{i}^{*}, i=1,2, \ldots, n$, such that $v_{i 1}\left(q_{i}^{*}, y^{*}, h\right)=0$.

Define

$$
\bar{q}_{i}=q_{i}-q_{i}^{*}, \bar{y}_{i}=y_{i}-y^{*}, \bar{x}_{i}=x_{i}-x_{i}^{*},
$$

then, system (4) is transformed into the following form

$$
\begin{aligned}
& \dot{\bar{q}}_{i}=\bar{v}_{i 1}\left(\bar{q}_{i}, \bar{y}_{i}, h_{i}^{\prime}\right), \\
& \dot{\bar{y}}_{i}=\bar{x}_{i}, \\
& \dot{\bar{x}}_{i}=\bar{v}_{i 2}\left(\bar{q}_{i}, \bar{y}_{i}, \bar{x}_{i}, h_{i}^{\prime}\right)+u_{i}-e^{*}+\bar{\theta}_{i},
\end{aligned}
$$

where $\bar{\theta}_{i}=\theta_{i}+v_{i 2}\left(q_{i}^{*}, y^{*}, x_{i}^{*}, h_{i}^{\prime}\right)+e^{*}, \bar{v}_{i 1}\left(\bar{q}_{i}, \bar{y}_{i}, h_{i}^{\prime}\right)=$ $v_{i 1}\left(\bar{q}_{i}+q_{i}, \bar{y}_{i}+y_{i}, h_{i}^{\prime}\right)-v_{i 1}\left(q_{i}^{*}, y^{*}, h_{i}^{\prime}\right), \bar{v}_{i 2}\left(\bar{q}_{i}, \bar{y}_{i}, \bar{x}_{i}, h_{i}^{\prime}\right)=$ $v_{i 2}\left(\bar{q}_{i}+q_{i}, \bar{y}_{i}+y_{i}, \bar{x}_{i}+x_{i}, h_{i}^{\prime}\right)-v_{i 2}\left(q_{i}^{*}, y^{*}, x_{i}^{*}, h_{i}^{\prime}\right), e^{*}$ is the steady state of the dynamic compensator $e_{i}$, which will be designed in the latter part, and it plays the role of eliminating the effect of the nonlinear term $v_{i 2}\left(q_{i}, y, x_{i}, h_{i}^{\prime}\right)$ on the optimal solution $y^{*}$, i.e., $v_{i 2}\left(q_{i}^{*}, y^{*}, x_{i}^{*}, h_{i}^{\prime}\right)+e^{*}=0$.

Assumption 4. There is a continuously differentiable function $V_{\bar{q}_{i}}\left(\bar{q}_{i}\right)$ that has lower and upper bounds by $\alpha_{1}\left(\bar{q}_{i}\right)$ and $\alpha_{2}\left(\bar{q}_{i}\right), i \in \mathcal{V}$, respectively, where $\alpha_{1}(\cdot)$ and $\alpha_{2}(\cdot)$ are two class- $\mathcal{K}_{\infty}$ functions. Moreover, its time derivative along the solution $\dot{\bar{q}}_{i}=\bar{v}_{i 1}\left(\bar{q}_{i}, \bar{y}_{i}, h_{i}^{\prime}\right)$ satisfies

$$
\dot{V}_{\bar{q}_{i}}\left(\bar{q}_{i}\right) \leq-\beta_{i}\left\|\bar{q}_{i}\right\|^{2}+\chi_{i}\left\|\bar{y}_{i}\right\|^{2}, \forall h_{i}^{\prime} \in W,
$$

where $\beta_{i}$ and $\chi_{i}$ are positive constants.

Remark 2. Assumption 3 is a necessary condition for obtaining the accurate optimal solution of DOSCP (5). This means that the state $y_{i}$ converges to $y^{*}$ if $q_{i}$ converges to $q_{i}^{*}, i=1,2, \ldots, n$. Assumption 4 describes the property of a minimum-phase nonlinear system, which can ensure the effectiveness of feedback control ( see Remark 3.10 in [27]). Since the transformed system (8) contains unmodeled dynamic part $\dot{\bar{q}}_{i}=\bar{v}_{i 1}\left(\bar{q}_{i}, \bar{y}_{i}, h_{i}^{\prime}\right)$, we first need Assumption 4 to guarantee that it is stable at the origin, then a distributed robust controller is designed such that the remaining parts of system (8) is stable at the origin. It is worth pointing out that this assumption is often used in the analysis and control of nonlinear systems. Therefore, Assumption 4 is a common and standard condition in the existing literatures, see [21], [22], [31].

\section{DISTRIBUTED OPTIMAL STATE CONSENSUS}

In view of existence of external disturbance, we need to design a robust control protocol to achieve the exact optimization rather than the solution of DOSCP (5) being converged into a bounded neighborhood of the optimal solution. Reviewing the description of external disturbance $\theta_{i}:=o\left(\nu_{i}, h_{i}^{\prime}\right)$, we know its form instead of its specific characteristics like eigenvalues. In what follows, we need to reconstruct the generator of external disturbance $\theta_{i}$ with linear observability. Specifically, the disturbance $\theta_{i}:=o\left(\nu_{i}, h_{i}^{\prime}\right)$ is polynomial in $\nu_{i}$, so is $\bar{\theta}_{i}$, according to Proposition 6.14 in [27], $\bar{\theta}_{i}=\theta_{i}+v_{i 2}\left(q_{i}^{*}, y^{*}, x_{i}^{*}, h_{i}^{\prime}\right)+e^{*}$ has the following minimal zeroing polynomial in $\nu_{i}$

$$
\rho_{i}(\lambda)=\lambda^{z_{i}}+\rho_{1} \lambda^{z_{i}-1}+\ldots+\rho_{z_{i}-1} \lambda+\rho_{z_{i}}
$$

for some real numbers $\rho_{1}, \rho_{2}, \ldots, \rho_{z_{i}-1}, \rho_{z_{i}}, h_{i}^{\prime} \in W$ and $z_{i} \in$ $\mathbb{R}^{+}$. Define $\omega_{i}=\left[\omega_{i 1}, \omega_{i 2}, \ldots, \omega_{i z_{i}}\right]^{T}$ with $\omega_{i 1}=\bar{\theta}_{i}, \omega_{i 2}=$ $\dot{\bar{\theta}}_{i}, \quad \ldots, \quad \omega_{i z_{i}}=\frac{d^{z_{i}-1} \bar{\theta}_{i}}{d t^{z_{i}-1}}, \quad i \in \mathcal{V}$, a generator of external disturbance $\bar{\theta}_{i}$ is established as

$$
\dot{\omega}_{i}=\Lambda_{i} \omega_{i}, \bar{\theta}_{i}=\Gamma_{i} \omega_{i}
$$

with

$$
\Lambda_{i}=\left[\begin{array}{c|c}
0 & I_{z_{i}-1} \\
\hline-\rho_{1} & -\rho_{2}, \ldots,-\rho_{z_{i}}
\end{array}\right], \quad \Gamma_{i}=\left[\begin{array}{c|c}
1 & 0_{1 \times z_{i}-1}
\end{array}\right] .
$$

Since the pair $\left(\Gamma_{i}, \Lambda_{i}\right)$ is observable, there exists a matrix $M_{i}$ such that the matrix $H_{i}=\Lambda_{i}+M_{i} \Gamma_{i}$ is Hurwitz. Then the linear dynamic compensator

$$
\dot{\eta}_{i}=H_{i} \eta_{i}+M_{i} u_{i}, \eta_{i}(0)=0
$$

is an internal model of (4), and $\eta_{i}$ is its state.

Define new coordinate and input transformation

$$
\bar{\eta}_{i}=\eta_{i}-\omega_{i}-M_{i} \bar{x}_{i}, \bar{u}_{i}=u_{i}+\Gamma_{i} \eta_{i}-e^{*},
$$

and using (7) again on system (4) leads to

$$
\begin{aligned}
& \dot{\bar{q}}_{i}=\bar{v}_{i 1}\left(\bar{q}_{i}, \bar{y}_{i}, h_{i}^{\prime}\right) \\
& \dot{\bar{y}}_{i}=\bar{x}_{i}, \\
& \dot{\bar{\eta}}_{i}=H_{i} \bar{\eta}_{i}+\tilde{v}_{i 2}\left(\bar{q}_{i}, \bar{y}_{i}, \bar{x}_{i}, h_{i}^{\prime}\right) \\
& \dot{\bar{x}}_{i}=\hat{v}_{i 2}\left(\bar{q}_{i}, \bar{y}_{i}, \bar{x}_{i}, h_{i}^{\prime}\right)+\bar{u}_{i}-\Gamma_{i} \bar{\eta}_{i},
\end{aligned}
$$

where

$$
\begin{aligned}
& \tilde{v}_{i 2}\left(\bar{q}_{i}, \bar{y}_{i}, \bar{x}_{i}, h_{i}^{\prime}\right)=H_{i} M_{i} \bar{x}_{i}-M_{i} \bar{v}_{i 2}\left(\bar{q}_{i}, \bar{y}_{i}, \bar{x}_{i}, h_{i}^{\prime}\right), \\
& \hat{v}_{i 2}\left(\bar{q}_{i}, \bar{y}_{i}, \bar{x}_{i}, h_{i}^{\prime}\right)=-\Gamma_{i} M_{i} \bar{x}_{i}+\bar{v}_{i 2}\left(\bar{q}_{i}, \bar{y}_{i}, \bar{x}_{i}, h_{i}^{\prime}\right) .
\end{aligned}
$$

With the help of coordinate transformations (7) and (13), it is observed from (14) that we can transform the distributed optimal state consensus problem (5) into a distributed stability problem with the internal model design. Thus we only need to design a distributed robust controller such that the converted problem is stabilizable at the origin. In turn, we can deduce that the original problem is solvable, and the optimal solution of the considered problem is further obtained.

For solving the distributed stabilization problem of system (14), the control protocol $\bar{u}_{i}$ in (14) to unit $i$ is designed as

$$
\begin{aligned}
& \bar{u}_{i}=-k \bar{x}_{i}-\phi g_{i}\left(\bar{y}_{i}\right)+\bar{e}_{i}+\bar{x}_{i}-\bar{\gamma}_{i}, \\
& \dot{\bar{\varphi}}_{i}=\sum_{j \in N_{i}}\left(\bar{y}_{i}-\bar{y}_{j}\right)+\sum_{j \in N_{i}}\left(\bar{x}_{i}-\bar{x}_{j}\right), \\
& \dot{\bar{e}}_{i}=-\bar{e}_{i}-\bar{x}_{i}-\hat{v}_{i 2}\left(\bar{q}_{i}, \bar{y}_{i}, \bar{x}_{i}, h_{i}^{\prime}\right)+\Gamma_{i} \bar{\eta}_{i},
\end{aligned}
$$

where $k$ and $\phi$ are positive constants to be determined, $\varphi_{i}$ and $e_{i}$ are the intermediate variables, $g_{i}\left(\bar{y}_{i}\right)=\nabla f_{i}\left(y_{i}\right)-$ $\nabla f_{i}\left(y^{*}\right), \bar{\gamma}_{i}=\sum_{j \in N_{i}}\left(\bar{y}_{i}-\bar{y}_{j}\right)+\sum_{j \in N_{i}}\left(\bar{x}_{i}-\bar{x}_{j}\right)+$ $\sum_{j \in N_{i}}\left(\bar{\varphi}_{i}-\bar{\varphi}_{j}\right), \bar{\varphi}_{i}=\varphi_{i}-\varphi_{i}^{*}, \bar{e}_{i}=e_{i}-e^{*}-\bar{x}_{i}, \varphi_{i}^{*}$ is the steady state of $\varphi_{i}, \nabla f_{i}\left(y_{i}\right)$ is the gradient of $f_{i}$ at $y_{i}$, the term $\sum_{j \in N_{i}}\left(\bar{y}_{i}-\bar{y}_{j}\right)+\sum_{j \in N_{i}}\left(\bar{x}_{i}-\bar{x}_{j}\right)$ is to realize 
consensus $\bar{x}_{i}=\bar{x}_{j},-\phi g_{i}\left(\bar{y}_{i}\right)$ is the negative gradient term to drive the deviation $\bar{y}_{i}$ converging to 0 , which implies that $y_{i}$ converges to the optimal solution $y^{*}$ of problem (5), $\sum_{j \in N_{i}}\left(\bar{\varphi}_{i}-\bar{\varphi}_{j}\right)$ is to eliminate the error of the consensus term.

Combining (14) with (15) leads to the closed-loop system

$$
\begin{aligned}
\dot{\bar{q}}_{i}= & \bar{v}_{i 1}\left(\bar{q}_{i}, \bar{y}_{i}, h_{i}^{\prime}\right), \\
\dot{\bar{y}}_{i}= & \bar{x}_{i}, \\
\dot{\bar{\eta}}_{i}= & H_{i} \bar{\eta}_{i}+\tilde{v}_{i 2}\left(\bar{q}_{i}, \bar{y}_{i}, \bar{x}_{i}, h_{i}^{\prime}\right), \\
\dot{\bar{x}}_{i}= & \hat{v}_{i 2}\left(\bar{q}_{i}, \bar{y}_{i}, \bar{x}_{i}, h_{i}^{\prime}\right)-k \bar{x}_{i}-\phi g_{i}\left(\bar{y}_{i}\right)-\Gamma_{i} \bar{\eta}_{i} \\
& +\bar{e}_{i}+\bar{x}_{i}-\bar{\gamma}_{i}, \\
\dot{\bar{\varphi}}_{i}= & \sum_{j \in N_{i}}\left(\bar{y}_{i}-\bar{y}_{j}\right)+\sum_{j \in N_{i}}\left(\bar{x}_{i}-\bar{x}_{j}\right), \\
\dot{\bar{e}}_{i}= & -\bar{e}_{i}-\bar{x}_{i}-\hat{v}_{i 2}\left(\bar{q}_{i}, \bar{y}_{i}, \bar{x}_{i}, h_{i}^{\prime}\right)+\Gamma_{i} \bar{\eta}_{i} .
\end{aligned}
$$

Lemma 2. Under Assumptions 1 and 3, the equilibrium point of (16) is the origin $(0,0,0,0,0,0)$ for any $i \in \mathcal{V}$.

Proof: Let $\left(\bar{q}_{i}^{0}, \bar{y}_{i}^{0}, \bar{\eta}_{i}^{0}, \bar{x}_{i}^{0}, \bar{\varphi}_{i}^{0}, \bar{e}_{i}^{0}\right)$ is the equilibrium point of (16), which satisfies

$$
\begin{aligned}
& 0=\bar{v}_{i 1}\left(\bar{q}_{i}^{0}, \bar{y}_{i}^{0}, h_{i}^{\prime}\right) \\
& 0=\bar{x}_{i}^{0} \\
& 0=H_{i} \bar{\eta}_{i}^{0}+\tilde{v}_{i 2}\left(\bar{q}_{i}^{0}, \bar{y}_{i}^{0}, 0, h_{i}^{\prime}\right) \\
& 0=\hat{v}_{i 2}\left(\bar{q}_{i}^{0}, \bar{y}_{i}^{0}, 0, h_{i}^{\prime}\right)-\phi g_{i}\left(\bar{y}_{i}^{0}\right)-\Gamma_{i} \bar{\eta}_{i}^{0}-\bar{\gamma}_{i}^{0}+\bar{e}_{i}^{0} \\
& 0=\sum_{j \in N_{i}}\left(\bar{y}_{i}^{0}-\bar{y}_{j}^{0}\right) \\
& 0=-\bar{e}_{i}^{0}-\hat{v}_{i 2}\left(\bar{q}_{i}^{0}, \bar{y}_{i}^{0}, 0, h_{i}^{\prime}\right)+\Gamma_{i} \bar{\eta}_{i}^{0},
\end{aligned}
$$

where $\bar{\gamma}_{i}=\sum_{j \in N_{i}}\left(\bar{y}_{i}^{0}-\bar{y}_{j}^{0}\right)+\sum_{j \in N_{i}}\left(\bar{\varphi}_{i}^{0}-\bar{\varphi}_{j}^{0}\right), \tilde{v}_{i 2}(\cdot)$, $\hat{v}_{i 2}(\cdot)$, and $g_{i}(\cdot)$ are defined in (14) and (15), respectively. According to Assumption 3, it is derived from (17a) that $\bar{q}_{i}^{0}=0$ and $\bar{y}_{i}^{0}=0$. It follows from (17c) that $\bar{\eta}_{i}^{0}=0$ since the matrix $H_{i}$ is Hurwitz. It is further derived from (17f) that $\bar{e}_{i}^{0}=0$. Then, for $(17 \mathrm{~d})$, we give

$$
0=\phi g_{i}\left(\bar{y}_{i}^{0}\right)-\sum_{j=1}^{n} a_{i j}\left(\bar{\varphi}_{i}^{0}-\bar{\varphi}_{j}^{0}\right) \text {. }
$$

Furthermore, $g_{i}\left(\bar{y}_{i}^{0}\right)=g_{i}(0)=0$, it follows from (18) and Assumption 1 that $\bar{\varphi}_{i}^{0}=\bar{\varphi}_{j}^{0}$, which implies that $\bar{\varphi}_{i}^{0}=\bar{\varphi}_{j}^{0}=$ $0, i \neq j=1,2, \ldots, n$. After the above analysis, it is known that the equilibrium point of (16) is the origin. The proof is complete.

Before performing subsequent analysis, let us check the structure of the distributed controller $u_{i}$ in system (4), which is designed as

$$
\begin{aligned}
& u_{i}=-k x_{i}-\phi \nabla f_{i}\left(y_{i}\right)-\gamma_{i}-\Gamma_{i} \eta_{i}+e_{i}, \\
& \dot{\varphi}_{i}=\sum_{j \in N_{i}}\left(y_{i}-y_{j}\right)+\sum_{j \in N_{i}}\left(x_{i}-x_{j}\right), \\
& \dot{e}_{i}=-k x_{i}-\phi \nabla f_{i}\left(y_{i}\right)-\gamma_{i}, \\
& \dot{\eta}_{i}=H_{i} \eta_{i}+M_{i} u_{i}, \eta_{i}(0)=0, i \in \mathcal{V},
\end{aligned}
$$

where $\gamma_{i}=\sum_{j \in N_{i}}\left(y_{i}-y_{j}\right)+\sum_{j \in N_{i}}\left(x_{i}-x_{j}\right)+$ $\sum_{j \in N_{i}}\left(\varphi_{i}-\varphi_{j}\right), k, \phi, \varphi_{i}$ and $e_{i}$ are the same as those in (15), $e_{i}$ is an offset term to eliminate the effect of the nonlinear term $v_{i 2}\left(q_{i}, y_{i}, x_{i}, h_{i}^{\prime}\right)$ on the first-order optimal condition, i.e., $e^{*}+v_{i 2}\left(q_{i}^{*}, y^{*}, x_{i}^{*}, h_{i}^{\prime}\right)=0$, and $-\Gamma_{i} \eta_{i}$ is an internal model term to compensate for an external disturbance. Concretely, (19) and (11) are plugged into (4) obtaining the closed-loop system under original coordinates in which $-\Gamma_{i}\left(\eta_{i}-\omega_{i}\right)$ converges to zero over time in the dynamics of $x_{i}$, only in this way, the trajectories of nonlinear system (4) converge accurately to the optimal solution of problem (5), and other terms play the same role as those in (15).

It is known from Lemma 3 that the equilibrium point of (16) is the origin. In what follows, our task is to find the appropriate parameters $\phi$ and $k$ to ensure the globally asymptotic stability of system (17) at the origin.

System (16) can be rewritten in a compact form

$$
\begin{aligned}
\dot{\bar{q}}= & \bar{v}_{1}(\bar{q}, \bar{y}, h) \\
\dot{\bar{y}}= & \bar{x} \\
\dot{\bar{\eta}}= & H \bar{\eta}+\tilde{v}_{2}(\bar{q}, \bar{y}, \bar{x}, h) \\
\dot{\bar{x}}= & \hat{v}_{2}(\bar{q}, \bar{y}, \bar{x}, h)-k \bar{x}-\phi g(\bar{y})-\Gamma \bar{\eta}+\bar{e}+\bar{x}, \\
& -\mathcal{L}(\bar{x}+\bar{\varphi}+\bar{y}) \\
\dot{\bar{\varphi}}= & \mathcal{L}(\bar{\varphi}+\bar{y}) \\
\dot{\bar{e}}= & -\bar{e}-\hat{v}_{2}(\bar{q}, \bar{y}, \bar{x}, h)-\bar{x}+\Gamma \bar{\eta}
\end{aligned}
$$

where $\bar{q}=\operatorname{col}\left(\bar{q}_{1}, \ldots, \bar{q}_{n}\right), \bar{y}, \bar{x}, \bar{\eta}, \bar{\varphi}, \bar{e}, g(\bar{y}), \bar{v}_{1}(\bar{q}, \bar{y}, h)$, $\tilde{v}_{2}(\bar{q}, \bar{y}, \bar{x}, h)$ and $\hat{v}_{2}(\bar{q}, \bar{y}, \bar{x}, h)$ are defined in the same way; $H=\operatorname{diag}\left\{H_{1}, H_{2}, \ldots, H_{n}\right\}$ and $\Gamma=\operatorname{diag}\left\{\Gamma_{1}, H_{2}, \ldots, H_{n}\right\}$. Define the following new coordinate transformations

$$
\sigma=E^{T} \bar{y}, \quad \vartheta=E^{T} \bar{x}, \quad \psi=E^{T} \bar{\varphi}, \varepsilon=E^{T} \bar{e},
$$

where $E=\left[\begin{array}{ll}E_{1} & E_{2}\end{array}\right]$ is an orthogonal matrix with $E_{1}=\frac{1}{\sqrt{n}} 1_{n} \in \mathbb{R}^{n}$ and $E_{2} \in \mathbb{R}^{n \times(n-1)}$ satisfying $E^{T} \mathcal{L} E=$ $\operatorname{diag}\left\{\hat{\lambda}_{1}, \hat{\lambda}_{2}, \ldots, \hat{\lambda}_{n}\right\}$ of which $0=\hat{\lambda}_{1}<\hat{\lambda}_{2} \leq \ldots \leq \hat{\lambda}_{n}$ are eigenvalues of $\mathcal{L}$ in an ascending order, $\sigma=\left[\sigma_{1}, \sigma_{2}^{T}\right]^{T}, \vartheta=$ $\left[\vartheta_{1}, \vartheta_{2}^{T}\right]^{T}, \psi=\left[\psi_{1}, \psi_{2}^{T}\right]^{T}, \varepsilon=\left[\varepsilon_{1}, \varepsilon_{2}^{T}\right]^{T}, \sigma_{1}, \vartheta_{1}, \psi_{1}, \varepsilon_{1} \in$ $\mathbb{R}$ and $\sigma_{2}, \vartheta_{2}, \psi_{2}, \varepsilon_{2} \in \mathbb{R}^{n-1}$. Thus, system (20) is decomposed into

$$
\begin{aligned}
\dot{\bar{q}}= & \bar{v}_{1}(\bar{q}, \bar{y}, h), \dot{\psi_{1}}=0, \dot{\sigma}_{1}=\vartheta_{1}, \dot{\sigma}_{2}=\vartheta_{2}, \\
\dot{\bar{\eta}}= & H \bar{\eta}+\tilde{v}_{2}(\bar{q}, \bar{y}, \bar{x}, h), \\
\dot{\vartheta}_{1}= & E_{1}^{T}\left(\hat{v}_{2}(\bar{q}, \bar{y}, \bar{x}, h)-\Gamma \bar{\eta}\right)-k \vartheta_{1}-\phi E_{1}^{T} g(\bar{y})+\vartheta_{1}+\varepsilon_{1}, \\
\dot{\vartheta}_{2}= & E_{2}^{T}\left(\hat{v}_{2}(\bar{q}, \bar{y}, \bar{x}, h)-\Gamma \bar{\eta}\right)-k \vartheta_{2}-\phi E_{2}^{T} g(\bar{y})+\vartheta_{2}+\varepsilon_{2} \\
& -E_{2}^{T} \mathcal{L} E_{2} \psi_{2}-E_{2}^{T} \mathcal{L} E_{2} \sigma_{2}-E_{2}^{T} \mathcal{L} E_{2} \vartheta_{2}, \\
\dot{\psi}_{2}= & E_{2}^{T} \mathcal{L} E_{2} \vartheta_{2}+E_{2}^{T} \mathcal{L} E_{2} \sigma_{2}, \\
\dot{\varepsilon}_{1}= & -\varepsilon_{1}-E_{1}^{T} \hat{v}_{2}(\bar{q}, \bar{y}, \bar{x}, h)-\vartheta_{1}+E_{1}^{T} \Gamma \bar{\eta}, \\
\dot{\varepsilon}_{2}= & -\varepsilon_{2}-E_{2}^{T} \hat{v}_{2}(\bar{q}, \bar{y}, \bar{x}, h)-\vartheta_{2}+E_{2}^{T} \Gamma \bar{\eta} .
\end{aligned}
$$

Due to $E_{1}=\frac{1}{\sqrt{n}} 1_{n}, \psi_{1} \equiv 0$ holds. Hence, we only need to analyze the stability of other variables in (22). The remaining variables are divided into three subparts, i.e. $\left(\sigma, \vartheta, \psi_{2}, \varepsilon\right), \bar{q}$ and $\bar{\eta}$ to analyze stability as follows.

First, for $\left(\sigma, \vartheta, \psi_{2}, \varepsilon\right)$, construct the function

$$
\begin{aligned}
V_{1}= & \frac{k}{2} \sigma_{1}^{T} \sigma_{1}+\frac{1}{2} \vartheta_{1}^{T} \vartheta_{1}+\sigma_{1}^{T} \vartheta_{1}+\frac{k}{2} \sigma_{2}^{T} \sigma_{2} \\
& +\frac{1}{2} \vartheta_{2}^{T} \vartheta_{2}+\sigma_{2}^{T} \vartheta_{2}+\frac{1}{2} \psi_{2}^{T} \psi_{2}+\frac{1}{2} \varepsilon^{T} \varepsilon .
\end{aligned}
$$


Clearly, when $k>1, V_{1}$ is positive.

Taking the time derivative of (23) along the solutions of (22) leads to

$$
\begin{aligned}
\dot{V}_{1} \leq & -(k-2)\|\vartheta\|^{2}-\hat{\lambda}_{2}\left\|\vartheta_{2}\right\|^{2}-\hat{\lambda}_{2}\left\|\sigma_{2}\right\|^{2} \\
& +(E \sigma+E \vartheta)^{T}\left(\hat{v}_{2}(\bar{q}, \bar{y}, \bar{x}, h)-\Gamma \bar{\eta}\right)-\phi(E \sigma)^{T} g(\bar{y}) \\
& -2 \vartheta_{2}^{T} E_{2}^{T} \mathcal{L} E_{2} \sigma_{2}-\phi(E \vartheta)^{T} g(\bar{y})-\|\varepsilon\|^{2}+\sigma^{T} \vartheta \\
& +\sigma^{T} \varepsilon-(E \varepsilon)^{T} \hat{v}_{2}(\bar{q}, \bar{y}, \bar{x}, h)+(E \varepsilon)^{T} \Gamma \bar{\eta},
\end{aligned}
$$

where $\hat{\lambda}_{2} I_{n-1} \leq E_{2}^{T} \mathcal{L} E_{2} \leq \hat{\lambda}_{n} I_{n-1}$ is used with $\hat{\lambda}_{2}$ and $\hat{\lambda}_{n}$ defined in Lemma 1 , and $E^{T} E=E E^{T}=I_{n}$.

According to Assumption 2, we have

$$
\begin{aligned}
& \phi(E \sigma)^{T} g(\bar{y})=\phi \bar{y}^{T} g(\bar{y}) \geq \phi m\|\sigma\|^{2}, \\
& \quad-\phi(E \vartheta)^{T} g(\bar{y})=-\phi \bar{x}^{T} g(\bar{y}) \leq \phi p\|\vartheta\|\|\sigma\| \\
& \leq \phi\left[p\left(\frac{p}{4 m}+1\right)\|\vartheta\|^{2}+\frac{p m}{p+4 m}\|\sigma\|^{2}\right],
\end{aligned}
$$

where $m=\min \left\{m_{1}, \ldots, m_{n}\right\}$ and $p=\max \left\{p_{1}, \ldots, p_{n}\right\}$.

In light of Lemma 7.8 in [27] and (21), the following inequality holds

$$
\begin{aligned}
& (E \sigma+E \vartheta)^{T}\left(\hat{v}_{2}(\bar{q}, \bar{y}, \bar{x}, h)-\Gamma \bar{\eta}\right)+(E \varepsilon)^{T} \Gamma \bar{\eta} \\
& -(E \varepsilon)^{T} \hat{v}_{2}(\bar{q}, \bar{y}, \bar{x}, h) \\
\leq & l_{1}\|\bar{q}\|^{2}+l_{2}\|\sigma\|^{2}+l_{3}\|\vartheta\|^{2}+l_{4}\|\bar{\eta}\|^{2}+\frac{1}{4}\|\varepsilon\|^{2}
\end{aligned}
$$

for positive scalars $l_{1}, l_{2}, l_{3}$ and $l_{4}$.

Combining (24), (25) with (26) yields

$$
\begin{aligned}
\dot{V}_{1} \leq & -(k-3)\|\vartheta\|^{2}-\hat{\lambda}_{2}\left\|\vartheta_{2}\right\|^{2}-\hat{\lambda}_{2}\left\|\sigma_{2}\right\|^{2}+\frac{5}{4}\|\sigma\|^{2} \\
& +\phi p_{0}\|\vartheta\|^{2}-\frac{4 \phi m^{2}}{p+4 m}\|\sigma\|^{2}+\hat{\lambda}_{n}\left\|\vartheta_{2}\right\|\left\|\sigma_{2}\right\| \\
& +l_{1}\|\bar{q}\|^{2}+l_{2}\|\sigma\|^{2}+l_{3}\|\vartheta\|^{2}+l_{4}\|\bar{\eta}\|^{2}-\frac{1}{2}\|\varepsilon\|^{2},
\end{aligned}
$$

where $p_{0}=p\left(\frac{p}{4 m}+1\right)$.

Letting

$$
V_{2}=\frac{1}{2}\left(\vartheta_{2}+\psi_{2}\right)^{T}\left(\vartheta_{2}+\psi_{2}\right),
$$

taking the time derivative of (28) along the solutions of (22) gives rise to

$$
\begin{aligned}
\dot{V}_{2}= & \left(E_{2} \vartheta_{2}+E_{2} \psi_{2}\right)^{T}\left(\hat{v}_{2}(\bar{q}, \bar{y}, \bar{x}, h)-\Gamma \bar{\eta}\right)-k\left\|\vartheta_{2}\right\|^{2} \\
& -\phi\left(E_{2} \vartheta_{2}\right)^{T} g(\bar{y})-\vartheta_{2}^{T} E_{2}^{T} \mathcal{L} E_{2} \psi_{2}-k \vartheta_{2}^{T} \psi_{2} \\
& -\phi\left(E_{2} \psi_{2}\right)^{T} g(\bar{y})-\hat{\lambda}_{2}\left\|\psi_{2}\right\|^{2} \\
\leq & d_{1}\|\bar{q}\|^{2}+d_{2}\|\sigma\|^{2}+d_{3}\|\vartheta\|^{2}+d_{4}\|\bar{\eta}\|^{2}-k\left\|\vartheta_{2}\right\|^{2} \\
& +\frac{1}{4}\left\|\vartheta_{2}\right\|^{2}+p^{2} \phi^{2}\|\sigma\|^{2}-\frac{1}{4} \hat{\lambda}_{2}\left\|\psi_{2}\right\|^{2}+\frac{p^{2} \phi^{2}}{\hat{\lambda}_{2}}\|\sigma\|^{2} \\
& +\frac{k^{2}}{\hat{\lambda}_{2}}\left\|\vartheta_{2}\right\|^{2}+\frac{\hat{\lambda}_{n}^{2}}{\hat{\lambda}_{2}}\left\|\vartheta_{2}\right\|^{2},
\end{aligned}
$$

where $d_{1}, d_{2}, d_{3}$ and $d_{4}$ are positive constants, the last inequality is obtained by using Young's Inequality, Lemma 7.8 in [27] and the second term of (25).

Second, we consider $\bar{q}$. The matrix $H_{i}$ is Hurwitz, so is the matrix $H$, then, there exists a symmetric and positivedefinite matrix $\Theta$ such that $\Theta H+H^{T} \Theta=-2 I$. Select the function

$$
V_{3}=\bar{\eta}^{T} \Theta \bar{\eta}
$$

and its time derivative along the solutions of (22) leads to

$$
\begin{aligned}
\dot{V}_{3} & =-2\|\bar{\eta}\|^{2}+2 \bar{\eta}^{T} \Theta \tilde{v}_{2}(\bar{q}, \bar{y}, \bar{x}, h) \\
& \leq-\|\bar{\eta}\|^{2}+\left\|\Theta \tilde{v}_{2}(\bar{q}, \bar{y}, \bar{x}, h)\right\|^{2} .
\end{aligned}
$$

According to Lemma 7.8 in [27], we have

$$
\begin{aligned}
\left\|\Theta \tilde{v}_{2}(\bar{q}, \bar{y}, \bar{x}, h)\right\|^{2} & \leq c_{1}\|\bar{q}\|^{2}+c_{2}\|\bar{y}\|^{2}+c_{3}\|\bar{x}\|^{2} \\
& =c_{1}\|\bar{q}\|^{2}+c_{2}\|\sigma\|^{2}+c_{3}\|\vartheta\|^{2},
\end{aligned}
$$

for positive constants $c_{1}, c_{2}$ and $c_{3}$.

Thus

$$
\dot{V}_{3} \leq-\|\bar{\eta}\|^{2}+c_{1}\|\bar{q}\|^{2}+c_{2}\|\sigma\|^{2}+c_{3}\|\vartheta\|^{2} .
$$

Third, consider $\bar{q}$. Let

$$
V_{4}=V_{\bar{q}}(\bar{q}),
$$

according to Assumption 4, and its time derivative along the solution of (22) yields

$$
\begin{aligned}
\dot{V}_{4}=\dot{V}_{\bar{q}}(\bar{q}) \leq & \sum_{i=1}^{n}\left(-\beta_{i}\left\|\bar{q}_{i}\right\|^{2}+\chi_{i}\left\|\bar{y}_{i}\right\|^{2}\right) \\
& =-\beta\|\bar{q}\|^{2}+\chi\|\bar{y}\|^{2} \\
& =-\beta\|\bar{q}\|^{2}+\chi\|\sigma\|^{2},
\end{aligned}
$$

where $\beta=\sum_{i=1}^{n} \beta_{i}, \chi=\sum_{i=1}^{n} \chi_{i}$ and $\|\bar{y}\|=\|E \sigma\|=\|\sigma\|$.

Finally, let $\bar{Z}=\left[\left\|\vartheta_{1}\right\|,\left\|\vartheta_{2}\right\|,\|\sigma\|,\left\|\psi_{2}\right\|,\|\bar{q}\|,\|\bar{\eta}\|,\|\varepsilon\|\right]^{T}$. For the whole system (22), take the Lyapunov function candidate

$$
V=V_{1}+b V_{2}+s V_{3}+\delta V_{4},
$$

where

$$
s=l_{4}+b d_{4}+1, \quad \delta=\frac{l_{1}+c_{1} s+b d_{1}+1}{\beta}
$$

and $b$ is a positive constant to be determined.

Let

$$
\begin{aligned}
\phi= & \frac{p+4 m}{3 m^{2}}\left(\frac{5}{4}+l_{2}+c_{2} l_{4}+c_{2}\right) \\
& +\frac{\chi(p+4 m)}{3 \beta m^{2}}\left(l_{1}+c_{1}\left(l_{4}+1\right)+1\right) .
\end{aligned}
$$

Combining (27), (29), (33) with (35) leads to

$$
\begin{aligned}
& \dot{V} \leq-\left[\begin{array}{lll}
\left\|\sigma_{2}\right\| & \left\|\vartheta_{2}\right\|
\end{array}\right] \mathbf{N}\left[\begin{array}{lll}
\left\|\sigma_{2}\right\| & \left\|\vartheta_{2}\right\|
\end{array}\right]^{T}-\|\bar{q}\|^{2} \\
& -\left(\left(k-3-l_{3}-\phi p_{0}-l_{4} c_{3}-c_{3}\right)-b b_{2}\right)\left\|\vartheta_{1}\right\|^{2} \\
& -\|\bar{\eta}\|^{2}-\frac{1}{4} \hat{\lambda}_{2}\left\|\psi_{2}\right\|^{2}-\left(1-b b_{0}\right)\left\|\vartheta_{2}\right\|^{2}-\frac{1}{2}\|\varepsilon\|^{2} \\
& -\left(\frac{\phi m^{2}}{p+4 m}-b b_{1}\right)\|\sigma\|^{2} \text {, }
\end{aligned}
$$

where $\mathbf{N}=\left[\begin{array}{cc}\hat{\lambda}_{2} & \frac{1}{2} \hat{\lambda}_{n} \\ \frac{1}{2} \hat{\lambda}_{n} & \mathbf{N}_{1}\end{array}\right], \mathbf{N}_{1}=k-4-\phi p_{0}-l_{3}-l_{4} c_{3}-c_{3}$, $b_{0}=d_{3}+\frac{k^{2}}{\hat{\lambda}_{2}}+\frac{1}{\hat{\lambda}_{2}} \hat{\lambda}_{n}^{2}+d_{4} c_{3}+\frac{1}{4}-k>0, b_{1}=d_{2}+p^{2} \phi^{2}+$ $\frac{p^{2} \phi^{2}}{\hat{\lambda}_{2}}+c_{2} d_{4}+\frac{\chi}{\beta}\left(c_{1} d_{4}+d_{1}\right)$ and $b_{2}=d_{3}+c_{3} d_{4}$.

Taking

$$
\begin{aligned}
& k>\max \left\{1, k_{1}, k_{2}\right\}, \\
& k_{1}=4+\phi p\left(\frac{p}{4 m}+1\right)+l_{4} c_{3}+c_{3}+\frac{\hat{\lambda}_{n}^{2}}{4 \hat{\lambda}_{2}}, \\
& k_{2}=3+\phi p\left(\frac{p}{4 m}+1\right)+l_{3}+l_{4} c_{3}+c_{3}, \\
& 0<b<\min \left\{\frac{k_{1}}{d_{3}+c_{3} d_{4}}, \frac{1}{b_{0}}, \frac{\phi m^{2}}{b_{1}(p+4 m)}\right\},
\end{aligned}
$$


it follows that $\mathbf{N}_{1}$ in (38) is nonnegative. According to Schur Complement Lemma, the matrix $\mathbf{N}$ is also nonnegative definite. It is further derived from (38) that

$$
\dot{V} \leq-Z^{T} \mathrm{O} Z
$$

with

$$
\mathrm{O}=\operatorname{diag}\left\{k_{1}-b b_{2}, 1-b b_{0}, \frac{\phi m^{2}}{p+4 m}-b b_{1}, \frac{b \hat{\lambda}_{2}}{4}, 1,1, \frac{1}{2}\right\} .
$$

Under (39), the matrix $\mathrm{O}$ is symmetric and positive-definite. It is derived from (40) that $\dot{V}$ is negative semi-definite. By virtue of the invariance principle (see Corollary 4.2 in [31]), it is concluded that system (22) is globally asymptotically stable at the origin. Furthermore, the variables $\bar{q}, \sigma, \psi$, $\vartheta, \varepsilon$ and $\bar{\eta}$ converge asymptotically to the origin, so are $\bar{x}, \bar{y}, \bar{\varphi}, \bar{q}, \bar{\eta}, \bar{e}$.

Therefore, the following theorem is obtained.

Theorem 1. Under Assumptions 1-4, given $\eta(0)=0$, algorithm (19) with the parameters $\phi$ and $k$ being respectively chosen as those in (37) and (39) solves the DOSCP (5).

Proof: With the help of the above analysis, it is proved that system (20) is globally asymptotically stable at the origin. Finally, it is obtained from Lemma 2 that the trajectories of system (4) converges to the optimal solution of the DOSCP (5) by the proposed algorithm (19) with the parameters $\phi$ and $k$ being chosen as those in (37) and (39), respectively. The proof is complete.

The lower and upper bounds of the function $V_{\bar{q}_{i}}\left(\bar{q}_{i}\right)$ in Assumption 4 are two class- $\mathcal{K}_{\infty}$ functions. Here its lower and upper bounds with respect to $\bar{q}_{i}$ is further assumed by a positive-definite quadratic function, respectively. Thus, the selected Lyapunov function candidate $V$ in (36) also has lower and upper bounds by $a_{1}\|Z\|^{2}$ and $a_{2}\|Z\|^{2}$ with $0<$ $a_{1} \leq a_{2}$, respectively. It follows that the closed-loop system (22) converges exponentially to the origin. Therefore, we get an important result under the following assumption.

Assumption 5. There is a continuously differentiable function $V_{\bar{q}_{i}}\left(\bar{q}_{i}\right)$ satisfying $r_{1}\left\|\bar{q}_{i}\right\|^{2} \leq V_{\bar{q}_{i}}\left(\bar{q}_{i}\right) \leq r_{2}\left\|\bar{q}_{i}\right\|^{2}$, $0<r_{1} \leq r_{2}$, and its derivative $\dot{V}_{\bar{q}_{i}}\left(\bar{q}_{i}\right)$ along $\dot{\bar{q}}_{i}=$ $\bar{v}_{i 1}\left(\bar{q}_{i}, \bar{y}_{i}, h\right)$ also satisfies the inequality (9).

Corollary 1. Under Assumptions 1-3 and 5, given $\eta(0)=0$, algorithm (19) with the parameters $\phi$ and $k$ being respectively selected according to (37) and (39) solves the DOSCP (5). Moreover, the convergence to the optimal solution $y^{*}$ is exponential.

Proof: Here, we only give a framework of the proof because its proof is very simple. First, select the Lyapunov candidate function (36), under Assumption 5, it is derived from Remark 3 that

$$
a_{1}\|Z\|^{2} \leq V \leq a_{2}\|Z\|^{2} .
$$

Second, similar to the proof of Theorem 1, taking the time derivative of (36) along the solutions of (22) yields (40), and selecting $\phi$ and $k$ according to (37) and (39), respectively, then under (39), combining (41) with (40) leads to $\|Z(t)\| \leq \sqrt{a_{2} / a_{1}}\|Z(0)\| e^{-\lambda_{\min }(\mathrm{O}) t /\left(2 a_{2}\right)}$ in which $\lambda_{\min }(\mathrm{O})$ is the minimum eigenvalue of the positive-definite matrix O. Third, it follows that the equilibrium point of system (22) is exponentially stable. Finally, it is obtained from Lemma 2 that the trajectories of system (4) driving by (19) converges exponentially to the optimal solution $y^{*}$, which implies that the convergence to the optimal solution $y^{*}$ is exponential. The proof is complete.

Remark 3. In [17]-[20], the dynamics of units are described by integral-type and linear systems, which are ideal systems. This means that the developed protocol may not be directly applied to the actual systems. The dynamics (4) of circuit units in this paper have practical physical models such as Chua's circuits and synchronous generators in the presence of external disturbances. There are few existing results on the optimal state consensus problems of such systems, and our results just fill this gap. Our proposed distributed optimization algorithm with a dynamic compensator (19) is used to offset the effect of the nonlinear term on the optimal solution of the problem. It can ensure that the trajectory of the algorithm converges to the optimal solution of the problem.

Remark 4. The disturbance is ubiquitous owing to the uncertainty and complexity of the working environment. The designed controllers in [17]-[20], [22] have no antidisturbance effect. As a result, the considered problem can not obtain an exactly optimal solution when an external disturbance exists. In contrast, the developed controller (19) in this paper has the accurately optimal solution to the problem (5) for system (4) with disturbance rejection due to the use of the internal model technology and gradient method.

Remark 5. Compared with the existing works [21], [34], this paper has no explicit restrictions on the initial states of the intermediate variables in the controller (19). However, in ( (17) in [21] and (21) in [34] ), they are required that the sum of the initial states of some variables are zero, i.e. $\sum_{i=1}^{n} \varsigma_{i}(0)=0$ in [21] and $\sum_{i=1}^{n} v_{i}(0)=0$ in [34].

Remark 6. We study the optimal state consensus problem of a multi-circuit system, where the system considered contains a small number of circuit units, and the information transmitted between neighbors is limited. This implies that the communication channels between neighbors should be sufficient for perfect information transmission. That is, there are no delay and congestion in the transmission of information. However, as the size of system increases, throughout and time-delay will have a serious impact on the exchange of information over the communication topology such as formation control of large-scale UAV systems and the economic dispatch problem of smart grids. This will be a research direction of our future work.

\section{DISTRIBUTED UNCERTAIN OPTIMAL STATE CON- SENSUS}

In Section 3, all units collaboratively solve the DOSCP with the fixed cost function (5). However, for many real cooperation problems [34], [35], the gradient of the local cost function cannot be accurately measured due to some 
unanticipated factors such as errors in measurements and stochastic noises from a vibration environment [34]. The class of distributed optimal state consensus problems has been widely studied such as online optimization [36], trajectory optimization [37], and so on. Particularly, the local cost function involves an uncertain term, namely,

$$
\begin{aligned}
& f(y, t)=\sum_{i=1}^{n} \bar{f}_{i}\left(y_{i}, t\right), \\
& \text { subject to }\left\|y_{i}-y_{j}\right\|=0,
\end{aligned}
$$

where $\bar{f}_{i}\left(y_{i}, t\right)=f_{i}\left(y_{i}\right)+\Delta_{i}(t), f_{i}\left(y_{i}\right)$ is the actual cost function, $\Delta_{i}(t)$ is the uncertain term generated by the evolution of (4), which is a bounded time-varying function. There is a significant difference between problems (5) and (42) that the cost function of (42) has a time-varying term $\Delta_{i}(t)$. This will induce some challenges for carrying out the global coordination tasks such as distributed algorithm design and construction of evaluation criteria for the optimal solutions. The regret function has become a performance evaluation criterion for developing algorithms in [38], [39], which has received wide attention. Its definition is the average difference between the real total cost and the ideal total cost resulting from the final state $y^{*}$ over a given time interval $[0, T]$ and $T>0$, i.e.

$$
R(0, T)=\frac{1}{T} \int_{0}^{T} \sum_{i=1}^{n}\left(\bar{f}_{i}\left(y_{i}, t\right)-\bar{f}_{i}\left(y^{*}, t\right)\right) d t
$$

To solve problem (42), the protocol $u_{i}$ in (19) is modified as

$$
\begin{aligned}
u_{i} & =-k x_{i}-\phi \nabla_{y_{i}} \bar{f}_{i}^{i n a}\left(y_{i}, t\right)-\gamma_{i}+e_{i}-\Gamma_{i} \eta_{i}, \\
\dot{\varphi}_{i} & =\sum_{j \in N_{i}}\left[\left(y_{i}-y_{j}\right)+\left(x_{i}-x_{j}\right)\right], \\
\dot{e}_{i} & =-k x_{i}-\phi \nabla_{y_{i}} \bar{f}_{i}^{i n a}\left(y_{i}, t\right)-\gamma_{i}, \\
\dot{\eta}_{i} & =H_{i} \eta_{i}+M_{i} u_{i}, \eta_{i}(0)=0, i \in \mathcal{V},
\end{aligned}
$$

where $\nabla_{y_{i}} \bar{f}_{i}^{i n a}\left(y_{i}, t\right)$ is the inaccurate gradient of $\bar{f}_{i}\left(y_{i}, t\right)$ $\left(\bar{f}_{i}\left(y_{i}, t\right)=f_{i}\left(y_{i}\right)+\Delta_{i}(t)\right)$, the parameters $\phi$ and $k$ also satisfy (37) and (39), respectively.

Remark 7. In the distributed uncertain optimal state consensus problem (42), the uncertain term $\Delta_{i}(t)$ is a bounded time-varying function. Define $\varpi_{i}=\nabla_{y_{i}} \bar{f}_{i}^{i n a}\left(y_{i}, t\right)-$ $\nabla f_{i}\left(y_{i}\right)$ as the gradient error, where $\nabla f_{i}\left(y_{i}\right)$ is the accurate gradient. We assume that the inexact gradient still satisfies Assumption 2 and its error is bounded. That is, there exist two positive constants $p_{i}$ and $\hat{\varpi}$ such that $\left\|\nabla_{s_{1}} \bar{f}_{i}^{i n a}\left(s_{1}, t\right)-\nabla_{s_{2}} \bar{f}_{i}^{i n a}\left(s_{2}, t\right)\right\| \leq p_{i}\left\|s_{1}-s_{2}\right\|$ and $\left\|\varpi_{i}\right\| \leq \hat{\varpi}, i=1,2, \ldots, n$.

Note that the control protocols (19) and (44) are the same except $u_{i}$ and the dynamic compensator $e_{i}$ owing to the inexact gradient. Hence, the difference between the closedloop systems in Sections 3 and 4 is the equation related to the gradient term. After the similar analysis and coordinate transformations as those in Section 3, and define $\bar{\varpi}=E^{T} \varpi$ with $\bar{\varpi}=\left[\bar{\varpi}_{1}, \bar{\varpi}_{2}^{T}\right]^{T}$ and $\varpi=\operatorname{col}\left(\varpi_{1}, \ldots, \varpi_{n}\right)$, the different parts of the closed-loop system in this section are given here in a decomposed form, and the same parts are omitted for simplicity (for details, please see (22))

$$
\begin{aligned}
\dot{\vartheta}_{1}= & E_{1}^{T}\left(\hat{v}_{2}(\bar{q}, \bar{y}, \bar{x}, h)-\Gamma \bar{\eta}\right)-k \vartheta_{1}-\phi\left(E_{1}^{T} g(\bar{y})+\bar{\varpi}_{1}\right) \\
& +\vartheta_{1}+\varepsilon_{1}, \\
\dot{\vartheta}_{2}= & E_{2}^{T}\left(\hat{v}_{2}(\bar{q}, \bar{y}, \bar{x}, h)-\Gamma \bar{\eta}\right)-k \vartheta_{2}-\phi\left(E_{2}^{T} g(\bar{y})+\bar{\varpi}_{2}\right) \\
& -E_{2}^{T} \mathcal{L} E_{2} \psi_{2}-E_{2}^{T} \mathcal{L} E_{2} \sigma_{2}+\vartheta_{2}+\varepsilon_{2} .
\end{aligned}
$$

Theorem 2. Under Assumptions 1-3 and 5, given $\eta(0)=0$, solutions $y_{i}$ of the uncertain DOSCP (42) converge exponentially to the optimal solution $y^{*}$ with a regret bound $R(0, T)$ by algorithm (44) with the parameters $\phi$ and $k$ being respectively chosen according to (37) and (39). Moreover, there exists a constant $e_{0}>0$ satisfying

$$
\begin{array}{r}
R(0, T) \leq \frac{6 \sqrt{n} e_{0} a_{2} z^{0}}{T \lambda_{\min }(\mathrm{O})} \sqrt{\frac{a_{2}}{a_{1}}}\left(1-e^{\left.-\frac{\lambda_{\min }(\mathrm{O})}{6 a_{2}} T\right)}\right. \\
+\sqrt{\frac{a_{2}}{a_{1}}} \frac{3 n \phi e_{0} \hat{\varpi}}{\lambda_{\min }(\mathrm{O})}
\end{array}
$$

with the matrix $\mathrm{O}$ defined in (40) and the initial error $z^{0}$ $=\sum_{i=1}^{n}\left(\left|q_{i}(0)\right|+\left|y_{i}(0)\right|+\left|x_{i}(0)\right|+\left|\varphi_{i}(0)\right|+\left|e_{i}(0)\right|\right)$.

Proof: Like the proof of Theorem 1, select the Lyapunov function candidate (36). Thus, we have

$$
\dot{V} \leq-\lambda_{\min }(\mathrm{O})\|Z\|^{2}+2 \phi\|Z\|\|\varpi\|
$$

where $\phi$ is the same as (37).

Owing to the boundedness of the error of the local gradient, it follows from (47) that $\dot{V} \leq-\frac{1}{3} \lambda_{\min }(\mathrm{O})\|Z\|^{2}$ holds for $\|Z\| \geq \frac{3 \sqrt{n} \phi \hat{\omega}}{\lambda_{\min }(\mathrm{O})}$. It is derived from Theorem 4.18 in [31] that $\|Z(t)\| \leq \sqrt{\frac{J\left(a_{2}\|Z(0)\|^{2}, t\right)}{a_{1}}}+\kappa(\hat{\varpi})$ for $t \geq 0$, where $J\left(J_{1}, J_{2}\right)=J_{1} \exp \left\{\frac{-\lambda_{\min }(\mathrm{O})}{2 a_{1} J_{2}}\right\}$ and $\kappa\left(\kappa^{*}\right)=\sqrt{\frac{a_{2}}{a_{1}}} \frac{3 \sqrt{n} \phi}{\lambda_{\min }(\mathrm{O})} \kappa^{*}$ in virtue of the proof of Lemma 4.4 and Theorem 4.9 in [31]. Therefore, we get $\|Z(t)\| \leq$ $\|Z(0)\| \sqrt{\frac{a_{2}}{a_{1}}} e^{-\frac{\lambda_{\min }(\mathrm{O})}{6 a_{2}} t}+\sqrt{\frac{a_{2}}{a_{1}}} \frac{3 \sqrt{n} \phi \hat{\omega}}{\lambda_{\min }(\mathrm{O})}, \forall t \geq 0$, which implies $\left\|y_{i}(t)\right\| \leq\left\|y^{*}\right\|+\sqrt{\frac{a_{2}}{a_{1}}}\left(\|Z(0)\|+\frac{3 \sqrt{n} \phi \hat{\omega}}{\lambda_{\min }(\mathrm{O})}\right), \forall t \in[0, T], T \geq$ 0 . According to Assumption 2, there is a positive constant $e_{0}>0, i \in \mathcal{V}$ such that

$$
\left\|\bar{f}_{i}\left(y_{i}, t\right)-\bar{f}_{i}\left(y^{*}, t\right)\right\| \leq e_{0}\left\|y_{i}-y^{*}\right\|, \forall t \in[0, T] .
$$

Hence, combining (6) with (48) leads to $R(0, T) \leq$ $\frac{\sqrt{n} e_{0}}{T} \int_{0}^{T}\|Z(t)\| d t$, which implies (46). Thus, the algorithm (45) solves the distributed uncertain state optimal consensus problem (42), and a linear regret bound is achieved. The proof is complete.

Remark 8. For the regret bound $R(0, T)$ in (46), its value is closely related to the initial error $z^{0}$, the gradient error's bound $\hat{\varpi}$ and the length of the integration interval $T$. If $z^{0}$ and $\hat{\varpi}$ are smaller, and $T$ is large enough, $R(0, T)$ will be smaller. In addition, the regret bound is also investigated in [38] and [39] for discrete-time systems with the inaccurate gradient, where the regret bounds are $O^{*}(\sqrt{n} / \sqrt{T})$ in [38] and $O^{*}\left(\sqrt{T}(\log T)^{\tilde{d}}\right)$ with $\tilde{d} \geq 1$ in [39]. It can be seen that the regret bounds are nonlinear caused by vanishing of step-size. However, in this paper, 


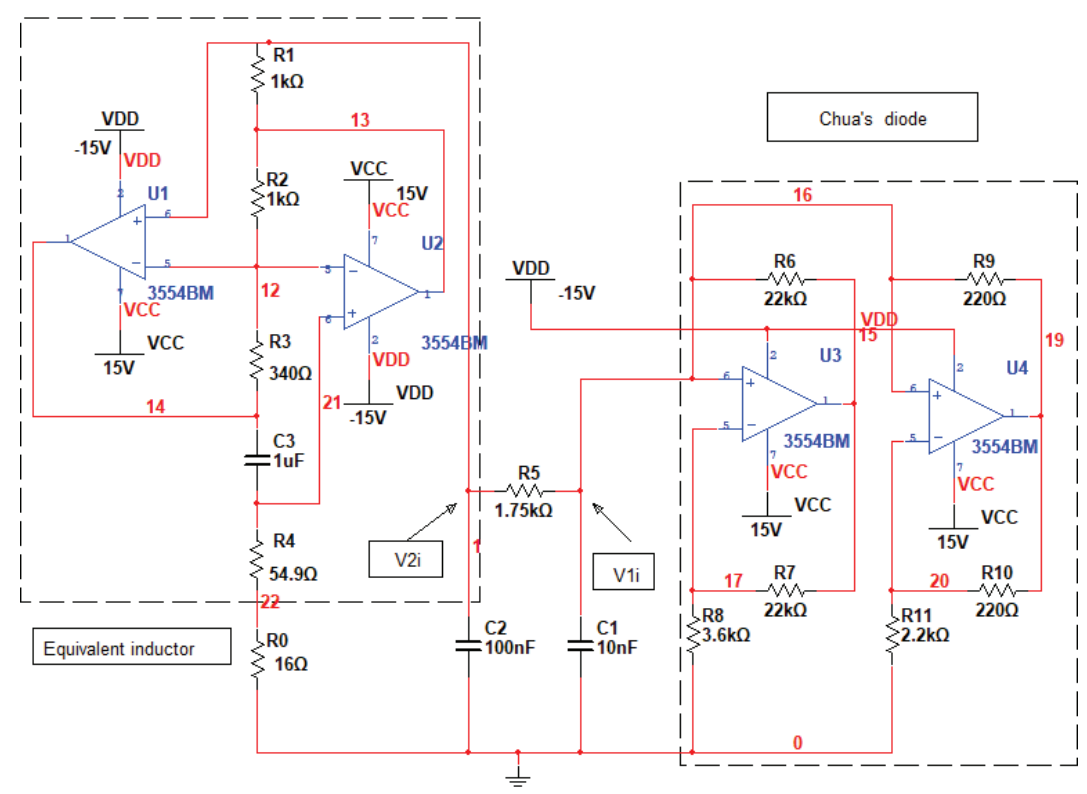

Fig. 3. The simulator of Chua's circuit $i$.

we obtain a linear regret bound $O^{*}(\sqrt{n} / T)$ for the distributed uncertain optimal consensus problem when the distributed algorithm with the fixed gains is employed.

Remark 9. The parameters on the upper bound of the regret bound $R(0, T)$ in (46) can be divided into parameters that are easy to determine and those that are difficult to determine. Specifically, the known parameters are the number of circuit units $n$, the initial error $z^{0}$ and the length of online time $T$. When the dynamics and the local cost function with uncertain term are given, and the optimal solution of the DOSCP is obtained, the parameters $\phi, e_{0}$ and $\hat{\varpi}$ are easily determined. However, the parameters $a_{1}, a_{2}$ and $\lambda_{\min }(\mathrm{O})$ are difficult to be determined since the parameter $b$ related to the Lyapunov function candidate (36) is not easily selected. Therefore, the upper bound of the regret bound $R(0, T)$ in real applications is not easily calculated. In this work, we know that the regret bound $R(0, T)$ is linear, which is an important result for determining the parameters in real applications.

Remark 10. Although the state consensus problem of nonlinear circuit systems has been studied in [6], [7], [9][11], [13], [15] using different methods, the energy consumption in each circuit unit is not taken into consideration in their consensus algorithms. Instead, this paper considers the minimization problem of energy consumption (modelled as the local cost function) when solving the state consensus problem for all the circuit units. Also the impact of external disturbances on the system performance is ignored in their works, but this paper takes it into consideration. Moreover, we prove that the proposed algorithm converges exponentially to the optimal solution of the problem under consideration with the help of some existing analysis methods, while the works mentioned above only proved that the states of circuits driven by the developed controller are con- sistent but its convergence rate is unknown. In addition, the distributed uncertain version is studied in this paper, and a linear regret bound induced by an inaccurate gradient of the local cost function is obtained..

\section{Simulation}

In this section, a state synchronization example is provided to verify the effectiveness of the proposed algorithms. The simulation study is performed in an electronic system consisting of 10 circuit units in the presence of external disturbances. The dynamics of each unit is modeled by (1). In experiments, we use the Multisim 10.0 software to build Chua's circuit. Suppose that there is no inductor that can be used, so a series of analog circuit components (resistors, operational amplifiers and capacitors) are used to construct an inductor equivalently. Moreover, the nonlinear part of Chua's circuit also needs to be equivalent. Specifically, the simulator of Chua's circuit $i$ is shown in Fig. 3. Note that, in the process of constructing the inductor and the nonlinear part of Chua's circuit, the value of the inductor parasitic resistance is negligible. In the case study, the parameters of each circuit unit are chosen as follows: $R_{0}=16 \Omega, L=L_{e q}=18.68 \mathrm{mH}, G_{a}=-0.41 \mathrm{mS}$, $G_{b}=-0.75 m S, C_{1}=10 n F, C_{2}=100 n F, R=1750 \Omega$ and $B_{0}=1 V$, which imply $d_{i 1}=10, d_{i 2}=-0.7175, d_{i 3}=$ $-1.3125, d_{i 4}=16.3945$ and $d_{i 5}=0.1499$. The communication topology among circuit units is described by an undirected and connected graph $G_{1}$ in Fig. 4, which implies that Assumption 1 is satisfied, and let the weight of each edge be 1 . As described in problem formulation of Section 2-B, all the circuit units need to find an agreement point as the estimate of known synchronized state by solving the optimal state consensus problem (5), where the local cost functions are $f_{1}\left(y_{1}\right)=\left(y_{1}+1\right)^{2}, f_{2}\left(y_{2}\right)=$ $2 y_{2}^{2}+3 y_{2}-4, f_{3}\left(y_{3}\right)=3 e^{0.5 y_{3}}, f_{4}\left(y_{4}\right)=0.5 e^{2 y_{4}}+7 y_{4}$, $f_{5}\left(y_{5}\right)=\frac{y_{5}^{2}+4}{\sqrt{y_{5}^{2}+2}}+y_{5}^{2}, f_{6}\left(y_{6}\right)=3 y_{6}^{2}+0.5 e^{2 y_{6}}+2, f_{7}\left(y_{7}\right)=$ 

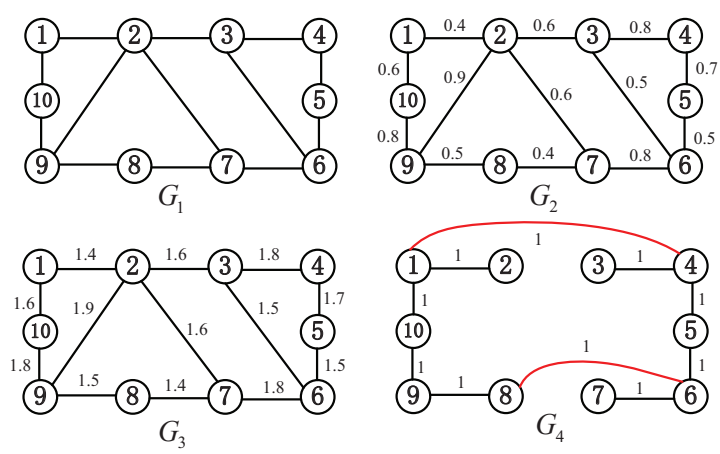

Fig. 4. The communication topology among ten circuit units.

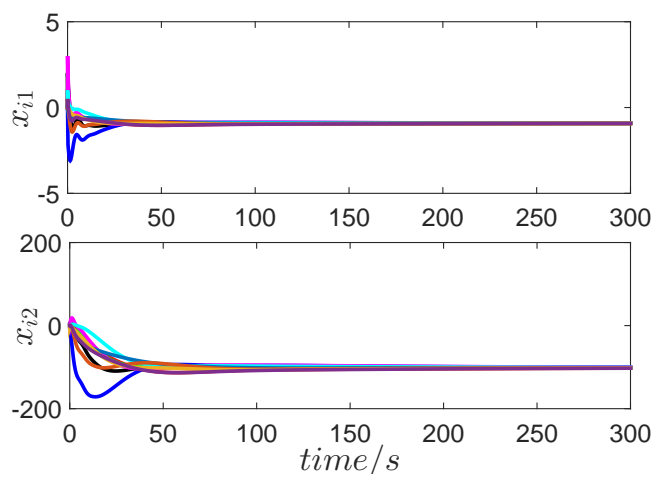

Fig. 5. Trajectories of $x_{i 1}$ and $x_{i 2}$ over $G_{1}, i=1,2, \ldots, 10$.

$4 y_{7}^{2}+6 y_{7}-10, f_{8}\left(y_{8}\right)=2 e^{0.3 y_{8}}+4 y_{8}, f_{9}\left(y_{9}\right)=2 y_{9}^{2}$ and $f_{10}\left(y_{10}\right)=y_{10}^{2}+4 y_{10}-10$. Each local function $f_{i}\left(y_{i}\right)$ is strongly convex, which implies that Assumption 2 is satisfied.

The external disturbance is set to be $\theta_{i}=A_{i} \sin \left(\varepsilon_{0} t+\hat{\gamma}\right)$ with $A_{i}=\frac{\sqrt{3}}{2} i$ and $\hat{\gamma}=\frac{\pi}{3}, i=1,2, \ldots, 10$. Clearly, $\theta_{i}$ is bounded. Let $\varepsilon_{0}=2$, it follows that the parameter matrices in (11) and (12) are obtained as $\Lambda_{i}=[0,2 ;-2,0], H_{i}=$ $[-5,2 ;-3,0], \Gamma_{i}=[1,0], M_{i}=[-5 ;-1]$. Let $\eta_{i}(0)=0$ and the initial states of other variables are arbitrarily chosen, $i=1, \ldots, 10$. The gains $k=30$ and $\phi=10$ are chosen in (19). It is observed from Fig. 5 that the states $x_{i 1}$ and $x_{i 2}$ of unit $i$ converge to $x_{i 1}^{*}=-0.9175$ and $x_{i 2}^{*}=-101.23$, respectively. Fig. 6 shows that the trajectories of $q_{i}$ and $e_{i}$ of circuit unit $i$ converge to constant $q_{i}^{*}=-102.1475$ and $e^{*}=-227.76$, respectively. In turn, let $y_{i}=x_{i 1}, x_{i}=-x_{i 1}+x_{i 2}-q_{i}$ and $h_{i}^{\prime}=\operatorname{col}\left(d_{i 1}, d_{i 2}, d_{i 3}, d_{i 4}, d_{i 5}\right)$, thus the problem (5) for nonlinear system (3) is transfomated into the DOSCP (5) for uncertain nonlinear state space equation (4). Then, we have $y^{*}=-0.9175$, which is the optimal solution of problem (5). It is observed from Fig. 7 that the state $x_{i}$ also converges to $x^{*}=0$. Based on the above analysis, it is demonstrated that the proposed algorithm (19) with an IM can solve the state synchronization problem for an electronic system with disturbance rejection.

In order to illustrate that the results we obtained are also applicable to the state synchronization problem of an electronic system whose communication topology is one of any undirected connected graphs, the following three cases are analyzed. Then we change the parameters $k$ and $\phi$ of the algorithm (19) to obtain some simulation results

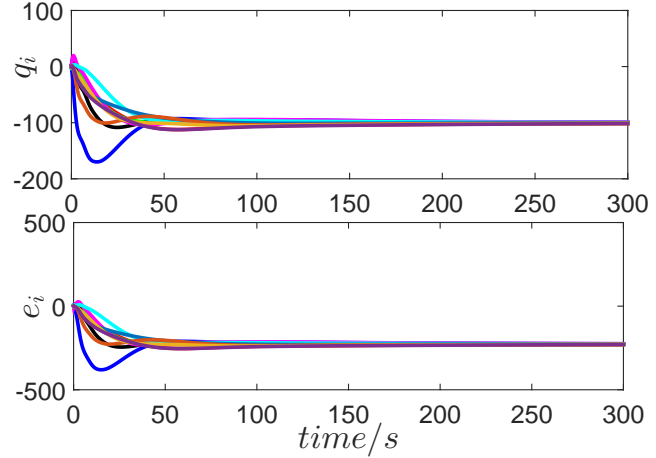

Fig. 6. Trajectories of $q_{i}$ and $e_{i}$ over $G_{1}, i=1,2, \ldots, 10$.

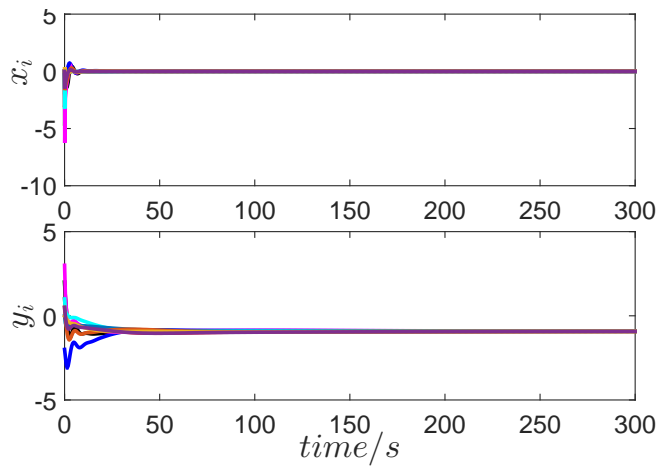

Fig. 7. Trajectories of $x_{i}$ and $y_{i}$ over $G_{1}, i=1,2, \ldots, 10$.

for statistical analysis to confirm the effectiveness of our proposed algorithm. Case 1): The communication topology is changed from $G_{1}$ to $G_{2}$ in Fig. 4. Compared with the network $G_{1}$, the weight of each edge in $G_{2}$ is unidentical, and the weight is less than 1; Case 2): The communication topology is changed from $G_{1}$ to $G_{3}$ in Fig. 4 in which the weight of each edge is the same and large than 1 ; and Case 3): The communication topology is changed from $G_{1}$ to $G_{4}$ in Fig. 4, where the graph $G_{4}$ adds the communication edges between units 1 and 4,6 and 8 , but removes the communication edges between units 2 and 3,2 and 7, 2 and 9,3 and 6,7 and 8 , and the weight of each edge is the same and equal to 1 . Due to the limited space, the simulation results are simply presented in Table 1 . The trajectories of states $x_{i 1}$ and $x_{i 2}$ of circuit $i$ over time are similar to Fig. 5, and they can converge to their optimal states respectively. Some conclusions can be drawn from Table 1: (a) If the electronic system has the same network structure, and the weights of the communication edges between units are the same, the states will converge to the optimal value more quickly when the weight of each edge becomes larger; (b) If the communication topology of the electronic system is inconsistent, but the weight of each edge is the same, the states will be synchronised more rapidly when the network has more connections; (c) No matter how the weights of the edges between units and the connectivity of the network change, as long as the communication topology of the electronic system is undirected and connected, the states can be synchronized; (d) When the parameter $\phi$ and the communication topology are unchanged, the states will be synchronised quickly with the decrease of the parameter $k$; 
TABLE 1

The relationship between synchronization time and algorithm parameters and communication topologies

\begin{tabular}{cccc}
\hline$k$ & $\phi$ & Communication topology & Synchronization time $(s)$ \\
\hline 30 & 10 & $G_{1}$ & 332 \\
30 & 10 & $G_{2}$ & 872 \\
30 & 10 & $G_{3}$ & 205 \\
30 & 10 & $G_{4}$ & 1403 \\
\hline 20 & 10 & $G_{1}$ & 236 \\
20 & 10 & $G_{2}$ & 491 \\
20 & 10 & $G_{3}$ & 173 \\
20 & 10 & $G_{4}$ & 834 \\
\hline 40 & 10 & $G_{1}$ & 412 \\
40 & 10 & $G_{2}$ & 925 \\
40 & 10 & $G_{3}$ & 309 \\
40 & 10 & $G_{4}$ & 1512 \\
\hline 30 & 15 & $G_{1}$ & 475 \\
30 & 15 & $G_{2}$ & 1160 \\
30 & 15 & $G_{3}$ & 268 \\
30 & 15 & $G_{4}$ & 1813 \\
\hline 30 & 5 & $G_{1}$ & 238 \\
30 & 5 & $G_{2}$ & 571 \\
30 & 5 & $G_{3}$ & 142 \\
30 & 5 & $G_{4}$ & 706 \\
\hline
\end{tabular}

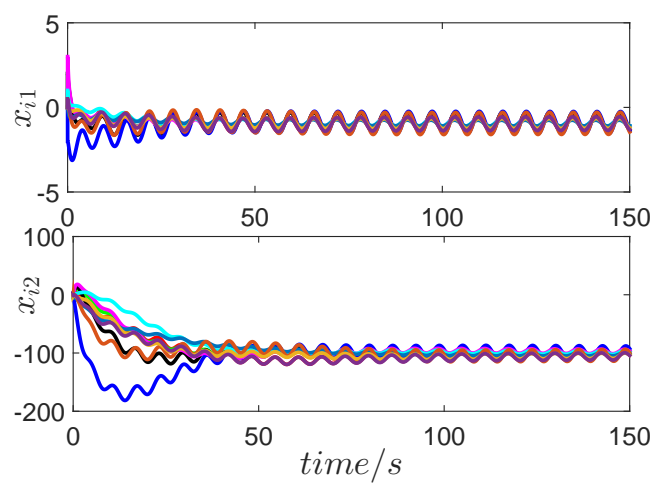

Fig. 8. Trajectories of $x_{i 1}$ and $x_{i 2}$ over $G_{1}, i=1,2, \ldots, 10$.

(e) When the parameter $k$ and the communication topology are unchanged, the states will be synchronised quickly with the decrease of the parameter $\phi$.

Next, consider an uncertain state synchronization problem example. The dynamics of each unit, the communication topology, the external disturbance, the parameters $k$ and $\phi$, the local cost function $f_{i}\left(y_{i}\right)$ and the initial states of variables are the same as those in the accurate state synchronization problem. The uncertain term is $\Delta_{i}(t)=$ $\sqrt{2} \sin \left(t+\frac{\pi}{4}\right), i=1,2, \ldots, 8$. Figs. 8 and 9 show that $x_{i 1}$, $x_{i 2}, q_{i}$ and $e_{i}$ converge to a regret bounded domains of $x_{i 1}^{*}$, $x_{i 2}^{*}, q_{i}^{*}$ and $e^{*}$, respectively. Similarly, under the coordinate transformations, it is observed from Fig. 10 that $x_{i}$ and $y_{i}$ also converge to a regret bounded neighborhoods of their respective optimal solutions.

Comparison: Here a comparison is provided to show the advantages of our algorithm. Specifically, the consensus problem of multiple Chua's circuit networks was studied in an ideal environment in [10], where the communication topology among the circuits is $G_{1}$ and the control protocol

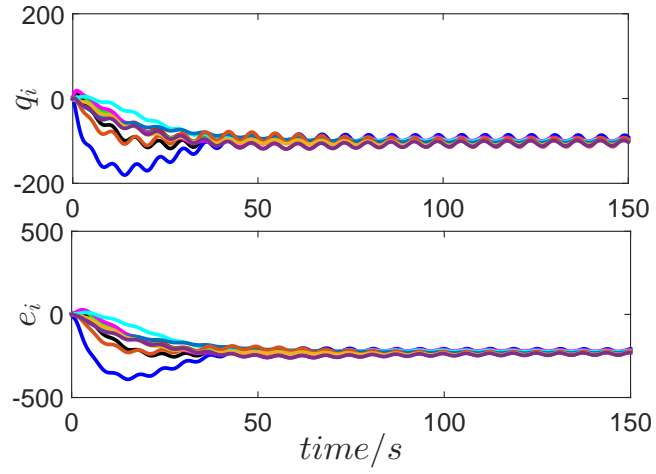

Fig. 9. Trajectories of $q_{i}$ and $e_{i}$ over $G_{1}, i=1,2, \ldots, 10$.

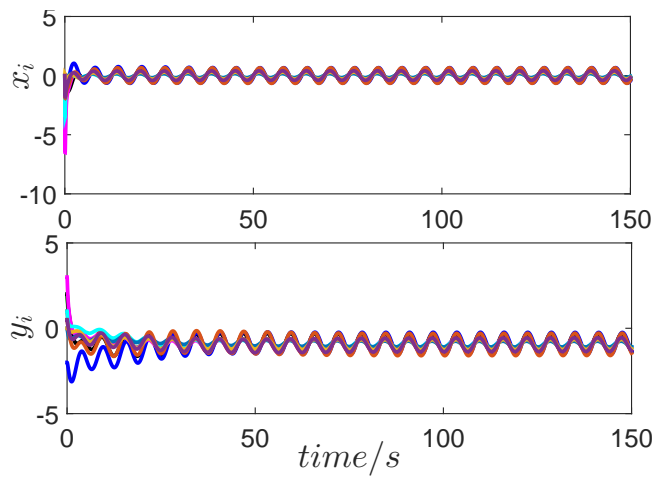

Fig. 10. Trajectories of $x_{i}$ and $y_{i}$ over $G_{1}, i=1,2, \ldots, 10$.

of each circuit $i$ is as follows

$$
u_{i}=-\sum_{j \in N_{i}}\left(y_{i}-y_{j}\right)-\sum_{j \in N_{i}}\left(x_{i}-x_{j}\right) .
$$

It can be observed from Fig. 11 that the states $x_{i 1}$ and $x_{i 2}$ of circuit $i$ converge to the origin. Let $y_{i}=x_{i 1}$ and $x_{i}=-x_{i 1}+x_{i 2}-q_{i}$, then it can be seen from Fig. 12 that $x_{i}$ and $y_{i}$ also converge to the origin. Furthermore, when considering the impact of the external environment on the system performance, the evolution curves of the states $x_{i 1}$ and $x_{i 2}$ of circuit $i$ over time are depicted in Fig. 13. Similarly, under the coordinate transformations, the trajectories of $x_{i}$ and $y_{i}$ are shown in Fig. 14, where the states converge to a neighborhood of the origin and the size of this neighborhood depends on the magnitude of the external disturbance. This implies that the algorithm in [10] is not robust. On the contrary, $x_{i 1}, x_{i 2}, x_{i}$ and $y_{i}$ in this paper converge to $x_{i 1}^{*}=-0.9175, x_{i 2}^{*}=-101.23, x^{*}=0$ and $y^{*}=-0.9175$ respectively rather than the origin because we not only need to achieve the state consistency but also ensure that the entire circuit system consumes minimal energy, that is, the consistent state is required to minimize the sum of a group of local cost functions. In addition, the proposed algorithm is robust to the external disturbance with a guaranteed convergence to the optimal solution.

\section{CONCLUSION}

In this paper, a novel continuous-time algorithm was proposed to cooperatively find the optimal solution of the distributed state consensus problem with disturbance rejection. It was proved that the proposed algorithms had the 


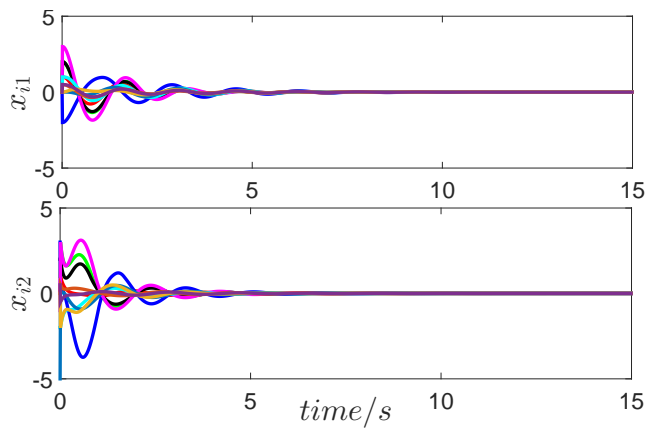

Fig. 11. Trajectories of $x_{i 1}$ and $x_{i 2}$ in [10], $i=1,2, \ldots, 10$.

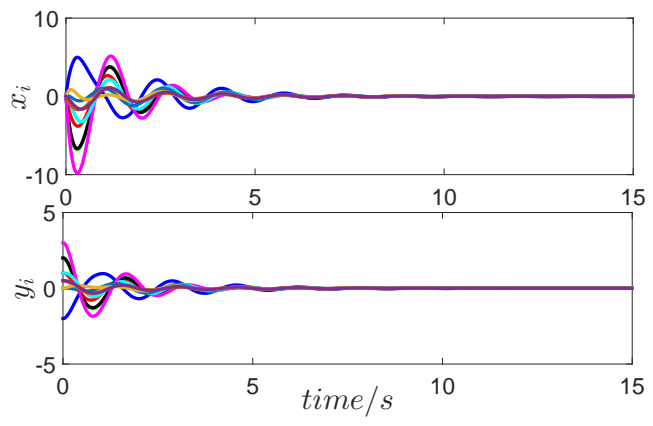

Fig. 12. Trajectories of $x_{i}$ and $y_{i}$ in [10], $i=1,2, \ldots, 10$.

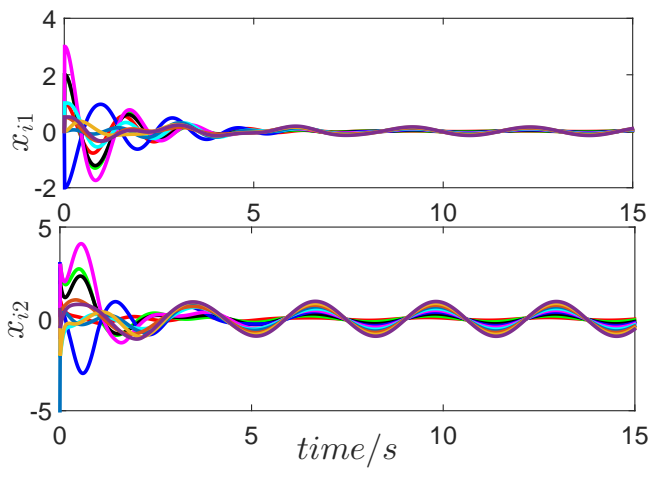

Fig. 13. Trajectories of $x_{i 1}$ and $x_{i 2}$ in [10], $i=1,2, \ldots, 10$.

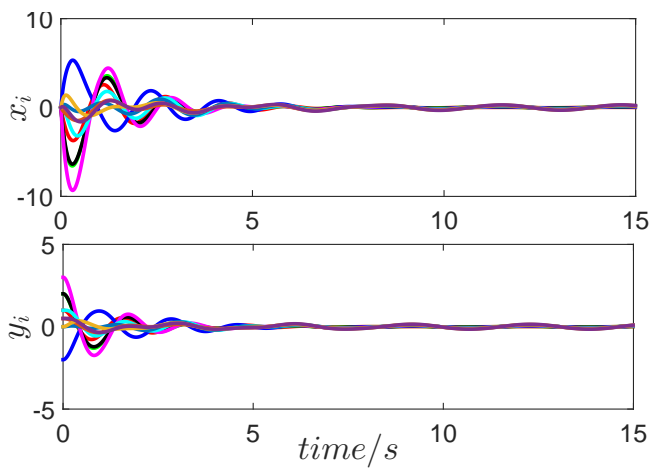

Fig. 14. Trajectories of $x_{i}$ and $y_{i}$ in [10], $i=1,2, \ldots, 10$. ability to guarantee that the trajectory of the considered system achieved consensus with a globally minimum cost. Numerical results indicated that our proposed algorithm can not only optimize the cost function but also reject external disturbance. Moreover, a linear regret bound was also obtained for the distributed uncertain optimal state consensus problem with the inexact gradient. Since the phase diagram of the Chua?s circuit has a double vortex structure, it can be applied to chaotic secure communication systems or spread spectrum communication systems. The proposed method can ensure that the states of the Chua?s circuit are not affected by the external environment, thus the proposed strategy provides a theoretical guarantee for the communication quality. When agents in a multi-agent system have the same dynamics as the Chua?s circuit, the proposed algorithm can also be applied to formation control problems of the multi-agent systems with disturbance rejection. Future work will continue to focus on the analysis and design of distributed methods for the optimal state consensus problem of multiple circuit systems over directed or time-vary graphs and will consider the distributed consensus problem subject to limited communication bandwidth or communication uncertainties.

\section{ACKNOWLEDGMENTS}

This work was supported in part by the National Natural Science Foundation of China under Grant 61973050 and Grant 61773089, by Fundamental Research Funds for the Central Universities under Grant DUT20GJ209 and Grant DUT20JC14.

\section{REFERENCES}

[1] X. Jin, D. Wei, W. He, L. Kocarev, Y. Tang, and J. Kurths, "Twistingbased finite-time consensus for Euler-Lagrange systems with an event-triggered strategy," IEEE Transactions on Network Science and Engineering, 2020.

[2] W. Ren and S. Nathan, "Distributed coordination architecture for multi-robot formation control," Robotics and Autonomous Systems, vol. 56, no. 4, pp. 324-333, 2008.

[3] D. Wang, Z. Wang, C. Wen, and W. Wang, "Second-order continuous-time algorithm for optimal resource allocation in power systems," IEEE Transactions on Industrial Informatics, vol. 15, no. 2, pp. 626-637, 2019.

[4] W. Du, W. Ying, P. Yang, X. Cao, G. Yan, K. Tang, and D. Wu, "Network-based heterogeneous particle swarm optimization and its application in uav communication coverage," IEEE Transactions on Emerging Topics in Computational Intelligence, vol. 4, no. 3, pp. 312-323, 2020.

[5] X. Shi, J. Cao, G. Wen, and X. Yu, "Finite-time stability for network systems with nonlinear protocols over signed digraphs," IEEE Transactions on Network Science and Engineering, 2020.

[6] H. Gang, L. Pivka, and A. Zheleznyak, "Synchronization of a one-dimensional array of chuaś circuits by feedback control and noise," IEEE Transactions on Circuits and Systems I: Fundamental Theory \& Applications, vol. 42, no. 10, pp. 736-740, 1995.

[7] M. T. Yassen, "Adaptive control and synchronization of a modified chuaś circuit system," Applied Mathematics \& Computation, vol. 135, no. 1, pp. 113-128, 2003.

[8] Q. Wei, X. Y. Wang, and X. P. Hu, "Chaos synchronization in complex oscillators networks with time delay via adaptive complex feedback control," Circuits Systems \& Signal Processing, vol. 33, no. 8, pp. 2427-2447, 2014.

[9] E. Sanchez, M. A. Matias, and V. Perez-Munuzuri, "Chaotic synchronization in small assemblies of driven chuaś circuits," IEEE Transactions on Circuits and Systems I: Fundamental Theory \& Applications, vol. 47, no. 5, pp. 644-654, 2000. 
[10] M. Magistris, M. Bernardo, E. Tucci, and S. Manfredi, "Synchronization of networks of non-identical chua's circuits: Analysis and experiments," IEEE Transactions on Circuits and Systems I: Regular Papers, vol. 59, no. 5, pp. 1029-1041, 2012.

[11] W. W. Chai, "Synchronization in arrays of coupled nonlinear systems with delay and nonreciprocal time-varying coupling," IEEE Transactions on Circuits \& Systems II: Express Briefs, vol. 52, no. 5, pp. 282-286, 2005.

[12] T. Zhang and G. Feng, "Output tracking of piecewise-linear systems via error feedback regulator with application to synchronization of nonlinear chuaś circuit," IEEE Transactions on Circuits and Systems I: Regular Papers, vol. 54, no. 8, pp. 1852-1863, 2007.

[13] A. Brambilla and G. S. Gajani, "Synchronization and small-signal analysis of nonlinear periodic circuits," IEEE Transactions on Circuits and Systems I: Regular Papers, vol. 55, no. 4, pp. 1064-1073, 2008.

[14] G. Wen, X. Yu, Z.-W. Liu, and W. Yu, "Adaptive consensusbased robust strategy for economic dispatch of smart grids subject to communication uncertainties," IEEE Transactions on Industrial Informatics, vol. 14, no. 6, pp. 2484-2496, 2018.

[15] A. Diwadkar and U. Vaidya, "Synchronization in large-scale nonlinear network systems with uncertain links," Automatica, vol. 100, pp. 194-199, 2019.

[16] H. Li, Q. Lu, and T. Huang, “Convergence analysis of a distributed optimization algorithm with a general unbalanced directed communication network," IEEE Transactions on Network Science and Engineering, vol. 6, no. 3, pp. 237-248, 2019.

[17] J. Tsitsiklis, D. Bertsekas, and M. Athans, "Distributed asynchronous deterministic and stochastic gradient optimization algorithms," IEEE Transactions on Automatic Control, vol. 31, no. 9, pp. 803-812, 1986.

[18] A. Nedic and A. Ozdaglar, "Distributed subgradient methods for multi-agent optimization," IEEE Transactions on Automatic Control, vol. 54, no. 1, pp. 48-61, 2009.

[19] J. Wang and N. Elia, "Control approach to distributed optimization," in Proceedings of Communication, Control, and Computing Conference. Allerton, IL, USA, 2010, pp. 557-561.

[20] S. S. Kia, J. Cortes, and S. Martinez, "Distributed convex optimization via continuous-time coordination algorithms with discretetime communication," Automatica, vol. 55, pp. 254-264, 2015.

[21] X. Wang, Y. Hong, and H. Ji, "Distributed optimization for a class of nonlinear multiagent systems with disturbance rejection." IEEE Transations on Cybernetics, vol. 46, no. 7, pp. 1655-1666, 2016.

[22] R. Li and G.-H. Yang, "Consensus control of a class of uncertain nonlinear multiagent systems via gradient-based algorithms," IEEE Transactions on Cybernetics, vol. 49, no. 6, pp. 2085-2094, 2018.

[23] S. Rahili and W. Ren, "Distributed continuous-time convex optimization with time-varying cost functions," IEEE Transactions on Automatic Control, vol. 62, no. 4, pp. 1590-1605, 2017.

[24] D. Yuan, D. W. Ho, and G.-P. Jiang, "An adaptive primal-dual subgradient algorithm for online distributed constrained optimization," IEEE Transactions on Cybernetics, vol. 48, no. 11, pp. 3045-3055, 2017.

[25] T. Yucelen and M. Egerstedt, "Control of multiagent systems under persistent disturbances," in Proceedings of American Control Conference. Montreal, QC, Canada, 2012, pp. 5264-5269.

[26] C. Wang, Z. Zuo, J. Sun, J. Yang, and Z. Ding, “Consensus disturbance rejection for lipschitz nonlinear multi-agent systems with input delay: a dobc approach," Journal of the Franklin Institute, vol. 354, no. 1, pp. 298-315, 2017.

[27] J. Huang, Nonlinear output regulation: theory and applications. SIAM, 2004, vol. 8 .

[28] R. T. Rockafellar, Convex analysis. NJ, USA: Princeton University Press, 2015.

[29] L. O. Chua, M. Itoh, L. Kocarev, and K. Eckert, "Chaos synchronization in chua's circuit," Journal of Circuits, Systems, and Computers, vol. 3, no. 1, pp. 93-108, 1993.

[30] Z. Zuo, Z. Lin, and Z. Ding, "Truncated prediction output feedback control of a class of lipschitz nonlinear systems with input delay," IEEE Transactions on Circuits and Systems II: Express Briefs, vol. 63, no. 8, pp. 788-792, 2016.

[31] H. K. Khalil, Nonlinear systems. NJ, USA: Prentice-hall, 2002.

[32] H. Bass, J. F. C. Kingman, F. Smithies, J. A. Todd, and C. T. C. Wall, Algebraic graph theory. Cambridge, U.K: Cambridge University Press, 1993.
[33] R. Olfati-Saber, J. A. Fax, and R. M. Murray, "Consensus and cooperation in networked multi-agent systems," Proceedings of the IEEE, vol. 95, no. 1, pp. 215-233, 2007.

[34] Y. Zhang, Z. Deng, and Y. Hong, "Distributed optimal coordination for multiple heterogeneous Euler-Lagrangian systems," Automatica, vol. 79, pp. 207-213, 2017.

[35] O. Devolder, F. Glineur, and Y. Nesterov, "First-order methods of smooth convex optimization with inexact oracle," Mathematical Programming, vol. 146, no. 1-2, pp. 37-75, 2014.

[36] S. Hosseini, A. Chapman, and M. Mesbahi, "Online distributed optimization via dual averaging," in Proceedings of 52nd IEEE Conference on Decision and Control. Florence, Italy, 2014, pp. 14841489.

[37] G. Foderaro, S. Ferrari, and T. A. Wettergren, "Distributed optimal control for multi-agent trajectory optimization," Automatica, vol. 50, no. 1, pp. 149-154, 2014.

[38] F. Yan, S. Sundaram, S. Vishwanathan, and Y. Qi, "Distributed autonomous online learning: Regrets and intrinsic privacypreserving properties," IEEE Transactions on Knowledge and Data Engineering, vol. 25, no. 11, pp. 2483-2493, 2013.

[39] N. Srinivas, A. Krause, S. M. Kakade, and M. W. Seeger, "Information-theoretic regret bounds for gaussian process optimization in the bandit setting," IEEE Transactions on Information Theory, vol. 58, no. 5, pp. 3250-3265, 2012.

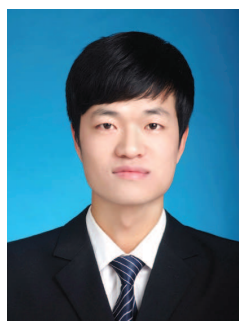

Zhu Wang received the B.Sc. degree from Harbin University of Science And Technology, Harbin, China, in 2016. Since 2016, He has been working toward the Ph.D. degree with the School of Control Science and Engineering, Dalian University of Technology, Dalian, China. His current research interests include distributed optimization, multi-agent systems and resource allocation in power systems.

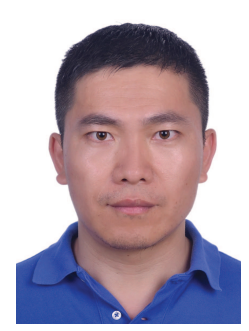

Dong Wang received the B.Sc. degree in automation and the M. Eng. degree in control theory and control engineering from the Shenyang University of Technology, Shenyang, China, in 2003 and 2006, respectively, and Ph.D. degree in control theory and control engineering from the Dalian University of Technology, China, in 2010. Since 2010, he has been with the Dalian University of Technology, where he is currently a professor with the School of Control Science and Engineering. His current research interests include multiagent systems, distributed optimization, fault detection and switched systems.

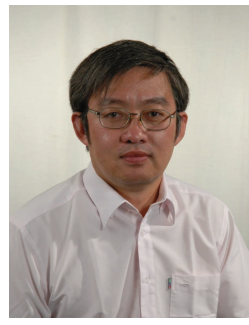

Dongbing Gu received the B.Sc. and M.Sc degrees in control engineering from the Beijing Institute of Technology, Beijing, China, and the Ph.D. degree in robotics from the University of Essex, Colchester, U.K. He was an Academic Visiting Scholar with the Department of Engineering Science, University of Oxford, Oxford, U.K., from 1996 to 1997. In 2000, he joined the University of Essex as a Lecturer, where he is currently a Professor with the School of Computer Science and Electronic Engineering. His current research interests include robotics, multiagent systems, cooperative control, model predictive control, visual SLAM, wireless sensor networks, and machine learning. 